Florida International University FIU Digital Commons

\title{
A computational method for internal radiation dosimetry at the voxel level
}

Swapna Chigurupati

Florida International University

DOI: 10.25148 /etd.FI14060800

Follow this and additional works at: https:// digitalcommons.fiu.edu/etd

Part of the Biomedical Engineering and Bioengineering Commons

\section{Recommended Citation}

Chigurupati, Swapna, "A computational method for internal radiation dosimetry at the voxel level" (2003). FIU Electronic Theses and Dissertations. 2330.

https://digitalcommons.fiu.edu/etd/2330

This work is brought to you for free and open access by the University Graduate School at FIU Digital Commons. It has been accepted for inclusion in FIU Electronic Theses and Dissertations by an authorized administrator of FIU Digital Commons. For more information, please contact dcc@fiu.edu. 


\section{FLORIDA INTERNATIONAL UNIVERSITY}

Miami, Florida

THE VOXEL LEVEL

A thesis submitted in partial fulfillment of the

requirements for the degree of

MASTER OF SCIENCE

in

BIOMEDICAL ENGINEERING

by

Swapna Chigurupati

2003 
To: Dean Vish Prasad

College of Engineering

This thesis, written by Swapna Chigurupati, and entitled A Computational Method for Internal Radiation Dosimetry at the Voxel Level, having been approved in respect to style and intellectual content, is referred to you for judgment.

We have read this thesis and recommend that it be approved.

Malcom Heimer

Ilker Yaylali

Juan Franquiz, Major Professor

Date of Defense: July 24, 2003

The thesis of Swapna Chigurupati is approved.

Dean Vish Prasad

College of Engineering

Dean Douglas Wartzok

University Graduate School

Florida International University, 2003 


\section{ACKNOWLEDGMENTS}

I wish to thank the members of the committee for their assistance and encouragement. I wish to express my sincere gratitude to my major professor, Dr.Juan Franquiz, for his continued support and for providing me with remarkable opportunities throughout my academic years at Florida international University. I want to thank my committee members, Dr. Malcolm Heimer and Dr. Ilker Yaylali for contributing their time and constant encouragement. I extend my gratitude to all of my friends for contributing their time and who inspired me throughout my master's degree. 


\section{ABSTRACT OF THE THESIS \\ A COMPUTATIONAL METHOD FOR INTERNAL RADIATION DOSIMETRY AT}

THE VOXEL LEVEL

by

Swapna Chigurupati

Florida International University, 2003

Miami, Florida

Professor Juan Franquiz, Major Professor

The current development of hybrid SPECT/CT and PET/CT systems allows not only accurate attenuation correction of images, but also provide an anatomical frame for the 3D spatial representation of the dose distribution. The main goal of this thesis project was to develop computational algorithms for calculation of the 3D dose distribution delivered by internal emitters based on the images and information provided by new hybrid SPECT/CT and PET/CT systems. Although many experimental problems exist in patientspecific dosimetry, current drawback is the lack of radionuclide voxel $\mathrm{S}$ values for the set of all possible combinations of cubical and non-cubical pixel edges and thickness used in SPECT and PET studies. This work presents an alternative and computationally efficient method for calculating voxel S values based on the Monte Carlo volume integration of tabulated dose point-kernels of beta emissions over a voxel-to-voxel geometry. The accuracy of the calculations was compared with those determined by direct Monte Carlo radiation transport simulation. 


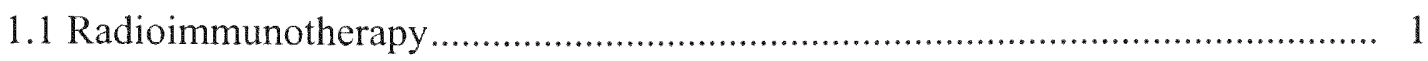

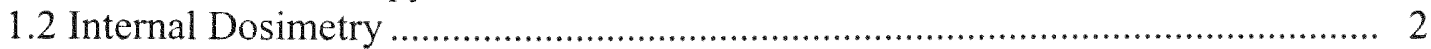

1.3Internal Dosimetry using the MIRD formalism................................................ 3

1.4 Significance of the Problem ............................................................................ 5

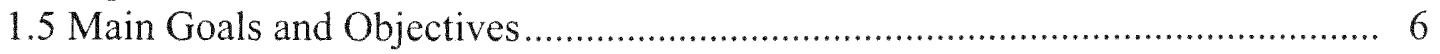

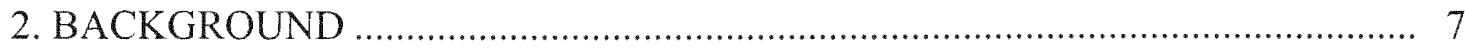

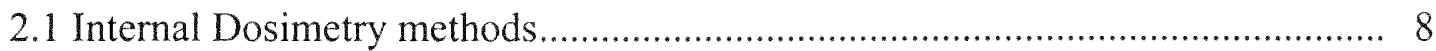

2.1.1 Basic Concepts ................................................................................. 8

2.1.2 The MIRD Formulation .................................................................. 8

2.2. Absorbed dose Calculation .............................................................. 11

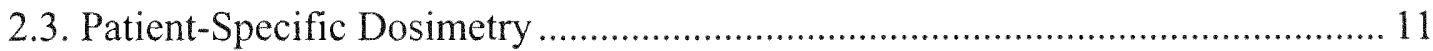

2.3.1.Motivation for patient-specific dosimetry ........................................... 11

2.3.2Activity Quantitation ........................................................................... 12

2.4 Absorbed dose calculation methods for Patient Specific Dosimetry .................... 14

2.4.1 Dose-Point Kernels ............................................................................. 14

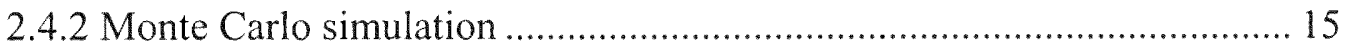

2.4.3.S Voxel Kernel method........................................................................ 16

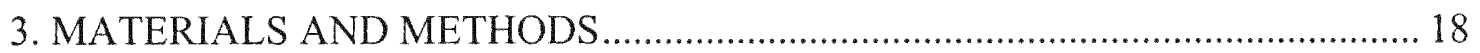

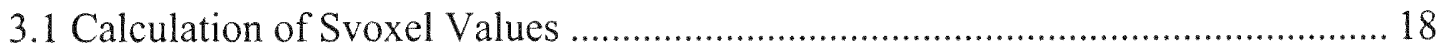

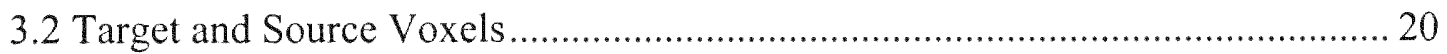

3.3 Random points in Source and Target voxel....................................................... 20

3.4 Explanation of Random number generations Program..................................... 21

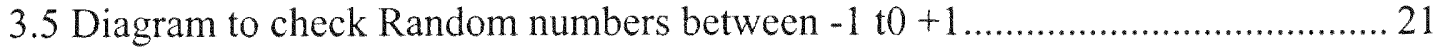

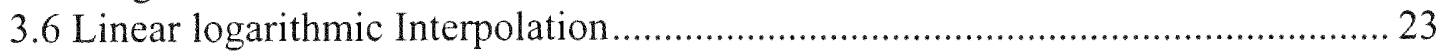

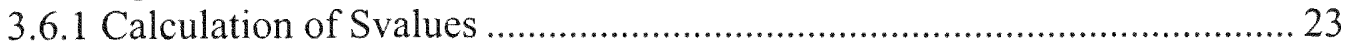

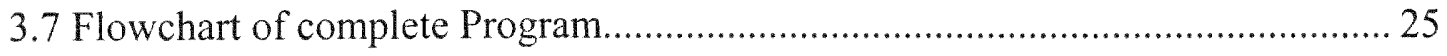

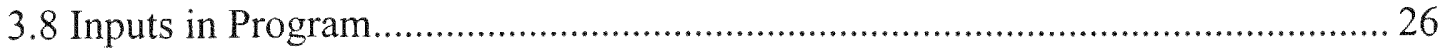

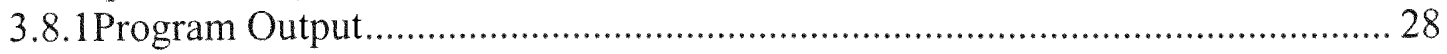

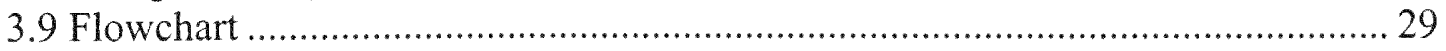

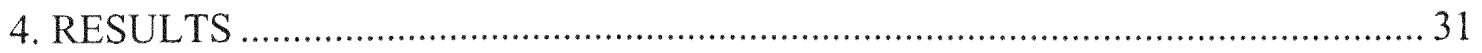




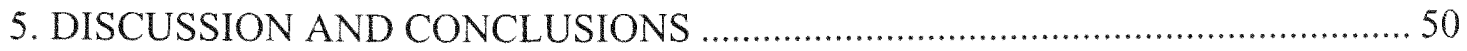

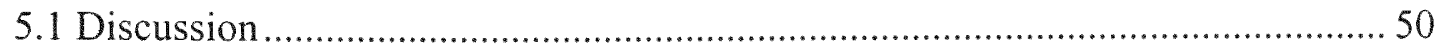

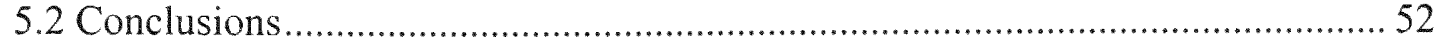

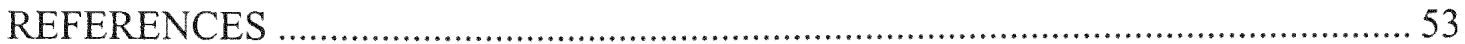

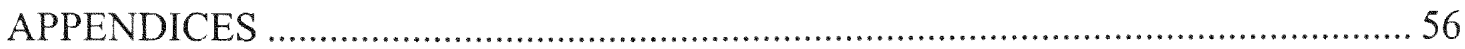




\section{LIST OF TABLES}

TABLE

PAGE

Table I: $\quad$ Voxel $\mathrm{S}$ values of cubical voxels $1 \mathrm{~mm}$ on edge........................................ 31

Table II Voxel S values of cubical voxels $2 \mathrm{~mm}$ on edge ….................................... 32

Table III: Voxel S values of cubical voxels $3 \mathrm{~mm}$ on edge ........................................ 33

Table IV: Voxel S values of cubical voxels $4 \mathrm{~mm}$ on edge........................................ 34

Table V: Voxel S values of cubical voxels $5 \mathrm{~mm}$ on edge …......................................35

Table VI: Voxel S values of cubical voxels $6 \mathrm{~mm}$ on edge ........................................ 36

Table VII: Voxel S values of cubical voxels $7 \mathrm{~mm}$ on edge ........................................3 37

Table VIII: Comparing Voxel Svalues of Radionuclide: P32 with voxel size $3 \mathrm{~mm}$ using Monte Carlo Volume Integration and Monte Carlo Radiation Transport Simulation method

Table IX: Comparing Voxel Svalues of Radionuclide: P32 with voxel size $6 \mathrm{~mm}$ using Monte Carlo Volume Integration and Monte Carlo Radiation Transport Simulation method

Table X: Comparing Voxel Svalues of Radionuclide: Sr89 with voxel size $3 \mathrm{~mm}$ using Monte Carlo Volume Integration and Monte Carlo Radiation Transport Simulation method

Table XI: Comparing Voxel Svalues of Radionuclide: Sr89 with voxel size $6 \mathrm{~mm}$ using Monte Carlo Volume Integration and Monte Carlo Radiation Transport Simulation method

Table XII: Comparing Voxel Svalues of Radionuclide: Y90 with voxel size $3 \mathrm{~mm}$ using Monte Carlo Volume Integration and Monte Carlo Radiation Transport Simulation method

Table XIII: Comparing Voxel Svalues of Radionuclide: Y90 with voxel size 3mm using Monte Carlo Volume Integration and Monte Carlo Radiation Transport Simulation method 


\section{LIST OF FIGURES}

FIGURE

Figure 1: Comparing Voxel Svalues of Radionuclide: P32 with voxel size $3 \mathrm{~mm}$ using Monte Carlo Volume Integration and Monte Carlo Radiation Transport Simulation method

Figure 2: Comparing Voxel Svalues of Radionuclide: P32 with voxel size $6 \mathrm{~mm}$ using Monte Carlo Volume Integration and Monte Carlo Radiation Transport Simulation method

Figure 3: Comparing Voxel Svalues of Radionuclide: $\mathrm{Sr} 89$ with voxel size $3 \mathrm{~mm}$ using Monte Carlo Volume Integration and Monte Carlo Radiation Transport Simulation method

Figure 4: Comparing Voxel Svalues of Radionuclide: $\mathrm{Sr} 89$ with voxel size $6 \mathrm{~mm}$ using Monte Carlo Volume Integration and Monte Carlo Radiation Transport Simulation method

Figure 5: Comparing Voxel Svalues of Radionuclide: Y90 with voxel size $3 \mathrm{~mm}$ using Monte Carlo Volume Integration and Monte Carlo Radiation Transport Simulation method

Figure 6: Comparing Voxel Svalues of Radionuclide: Y90 with voxel size $6 \mathrm{~mm}$ using Monte Carlo Volume Integration and Monte Carlo Radiation Transport Simulation method 


\section{INTRODUCTION}

1.1 Radioimmunotherapy (RIT) is a new area of medicine being studied to locate and treat cancer within the body using Radioisotopes. RIT uses a radioactive material attached to specially designed antibodies with internal or other compounds to locate and treat cancer within the body. Antibodies are naturally produced by the body's immune system. They are normally used to recognize infections caused by bacteria and viruses. The antibodies used in RIT are monoclonal antibodies (MAbs). These antibodies are developed in the laboratory and recognize substances on the surface of tumor cells. The MAbs are modified to bind radioactive metals (Indium-111 or Iodine-123), which can be visualized with a special camera in Nuclear Medicine. Images from this camera show areas where the MAbs have localized in the body. RIT uses the same MAbs for therapy but switches the radioactive metal to Yttrium-90 which delivers local radiation to the tumor. The radiolabeled $\mathrm{MAb}$ is administered through a vein and then circulates through the body to the surface of tumor cells. The tumor cells are destroyed by the radiation given off from the localized radiolabeled MAbs.

Radionuclides are administered to patients in nuclear medicine procedures in a variety of diagnostic and therapeutic applications. A key consideration in such studies is the absorbed dose to different organs of the patient. This concern is naturally heightened in therapy applications, where a significant absorbed dose may be received by other organs and in particular by radiosensitive organs.

Radionuclide therapy based on patient-specific dosimetry offers the potential for optimizing the dose delivered to the target tumor through utilization of measured radiopharmaceutical kinetics specific to the individual. The administered activity may be 
tailored for the patient such that the highest possible radiation dose may be given to the tumor while limiting the dose to critical organs and tissues below any designated threshold for negative biological effects.

1.2 Internal Dosimetry: It is the scientific methodology used to measure, calculate, estimate, assay, predict and otherwise quantify the radioactive energy absorbed by the ionization and excitation of atoms in human tissues as a result of the emission of energetic radiation by internally deposited radio nuclides. Internal Dosimetry deals with the determination of the amount and the spatial and temporal distribution of radiation energy deposited in tissue by radionuclides within the body. Internal dose is calculated from external measurements of activity in the patient.

Methods for acquiring quantitative data on radionuclide biodistribution and or calculation of the radiation absorbed dose using standard anthropomorphic models were developed by the Medical Internal Radiation Dose (MIRD) committee of the Society of Nuclear Medicine. This system of mathematical equations for calculating the internal dosimetry of administered radiopharmaceuticals is referred to as the MIRD schema. Computer software tools such as MIRDOSE are available for implementing the source-organ residence times that may be obtained from imaging and analysis of time activity curves. The MIRD schema were originally designed to provide conservative estimates of absorbed dose for diagnostic radiopharmaceuticals. The MIRD schema have also been used to estimate organ doses for therapeutic radioimmunoconjugates and other radioactive drugs (Darrell, 2000). Patient specific dosimetry refers to the estimation of radiation dose to tissues of a specific patient, based on his or her individual body habitus 
and measured radiopharmaceutical kinetics rather then on an average anthropomorphic model and hypothetic kinetics. In contrast to the average dose, position-specific dosimetry refers to radiation doses to specific points in a tumor or organ and thus reflects the spatial variation in dose within a target tissue (Zanzonocio, 2000). More recently, MIRD pamphlet no. 17, the Dosimetry of Nonuniform Activity Distributions: Radionuclide $S$ values at the Voxel Level has extended the MIRD schema to arbitrary macroscopic activity distributions in 3 dimensions for calculation of the resulting macroscopic dose distribution.

\subsection{Internal Dosimetry using the MIRD formalism}

Absorbed dose calculations require determination of patient pharmacokinetic data using a diagnostic activity prior to the administration of the larger therapeutic activity.

Since there is no practical or accepted method for patient-specific internal dose calculation, the formalism of the Medical Internal Dose Committee (MIRD) of the Society of Nuclear Medicine has been used for dose calculation in most clinical trials where the radiopharmaceutical does not distribute uniformly. The MIRD formalism developed for radiation safety purposes in diagnostic nuclear medicine studies, calculates organ doses per unit of activity by using a diagnostic tracer activity prior to the treatment. This formalism is based on the use of radionuclide specific absorbed fractions, or $\mathrm{S}$ values, defined as the mean absorbed dose to a target organ per radioactive decay in a source organ. The average target organ dose is given by the product of the source organ cumulated activity and the corresponding $\mathrm{S}$ value. Cumulated activity is the time integral of the activity in the source organ. The total target organ dose is the summation of all 
source organ contributions. The activity in each source organ at different time-points is determined from regions of interest on the geometric mean of a gamma camera whole body anterior and posterior conjugate opposite views. Radionuclide $\mathrm{S}$ values have been derived from MC simulation of radiation transport in standard anthropomorphic models and assuming a uniform organ activity distribution.

Although the value of MIRD formalism for calculating average internal doses in nuclear medicine diagnostic studies, this model has three major conceptual limitations to be used in internal radiotherapy treatment planning. The first limitation is that tumor and metastases, which uptake a significant amount of the radiopharmaceutical, are not included in the model. Second, the MIRD standard anatomic models do not fit the real dimensions and morphology of each patient. The critical effect of organ morphology and sizes in dosimetry calculations has been demonstrated by several studies (Clairand et al., 2000; Breitz, 2002). The third limitation is the assumption of uniform activity and dose in organs does not allow considering the dosimetry effect of cold spots on the tumor and hot spots in normal organs. It is known that the non-uniform distribution of therapeutic dose significantly affects the regression/ablation in the case of tumors and the radiotoxicity of normal tissues (Furhang et al., 1997).

In addition absorbed dose delivered by beta particles is calculated by assuming local absorption at the source organs and no consideration is given to the range of beta particles. Attempt to adapt the MIRD formalism to a more patient-specific approach have included the simulation of tumors by MIRD standard organs (Leichner and Kwok, 1993), estimation of tumor volume by CT and use of S sphere values (Wessels et al., 1990), and the MABDOSE (Johnson et al., 1999a; 1999b) and DOSE3D formalisms including 
spherical tumors, MC dose calculations and more flexibility in organ sizes and locations. However, the basic limitations of standard anatomic geometries and uniform organ activity and dose remain in all these approaches.

In addition to the conceptual limitations described above, the quantization of the whole body anterior and posterior views required for the MIRD formalism is affected by significant experimental errors, such as the attenuation and scattering of photons, tissue background subtraction, overlapping of organs, lack of organ visualization in one of the views and no information on organ masses. All these errors and model limitations make the inaccuracy of MIRD calculations.

\subsection{Significance of the Problem}

At present, two methods can satisfy patient-specific dosimetry requirements for internal radiotherapy. These methods are the Monte Carlo simulation of radiation transport based dosimetry and the 3D convolution of the cumulative activity with a dose-point kernel radial function. MC main drawback is the extended computational time, these are timeintensive computations.

Dose-point kernels are radially symmetric functions representing the absorbed dose in a target point per radioactive decay in a source point. These functions have been generated for Monte Carlo radiation transport simulations for an unbounded homogeneous medium, usually water (Cross et al., 1992; Furhang et al., 1996) and represented by analytical functions for beta (Preswitch et al., 1989) and gamma emissions (Furhang et al., 1996). The cumulative activity to be convolved is derived from the SPECT or PET slices (Erdi et al., 1998). The main problem of the convolution is that is difficult incorporating tissue 
inhomogenities since the voxel $\mathrm{S}$ values can only be generated assuming an infinite, homogeneous medium. A second difficulty is the election of the grid size and how to define the source and the target. Nuclear image data are acquired in a coarse matrix and there is not much sense to carry out the dose calculations on such a fine scale. The voxel source kernel is the method that considers the experimental limit of resolution at which nuclear images are acquired and reconstructed.

The overall goal of this project is improved patient-specific radiotherapy planning using internal emitters. To accomplish this goal, an internal dosimetry calculation protocol based on hybrid SPECT/CT images, Monte Carlo simulation and a dose-voxel kernel convolution method will be developed, validated and implemented as a software toolbox for its clinical and research use.

One current limitation of dosimetry calculations at the voxel level using the convolution method is the lack of voxel S values for different radionuclides and voxel sizes.

\subsection{Main Goals and Objectives}

To develop algorithms and software tools for 3D absorbed dose distributions using emission and transmission images.

For accomplishing these objectives and to compensate the lack of S values, we developed and implemented, a simple algorithm for determining voxel $\mathrm{S}$ values based on the Monte Carlo integration of dose-point kernels on cubical source-target geometry in $\mathrm{C}++$ Software.

And these Results were compared with those of the Bolch method. 


\section{BACKGROUND}

Radionuclides are administered to patients in nuclear medicine procedures in a variety of diagnostic and therapeutic applications. A key consideration in such studies is the absorbed dose to different organs of the patient; this concern is naturally heightened in therapy applications, where a significant absorbed dose may be received by other organs and in particular by radiosensitive organs. Absorbed doses are calculated with the aid of anthropomorphic phantoms, i.e. mathematical representations of the human body, which provide the absorbed fractions and organ masses. A valid internal dose estimate depends heavily on the collection of kinetic data for organs which concentrate the radiopharmaceutical (source organs), the whole body and for all excretion pathways. Obtaining these data require the proper measurement methods and acquisition of data at appropriate time points. The information obtained in a dosimetry study is used in many different ways, including evaluation of individual trials, and in the approval of radioactive drugs for general use. Radiation dose estimates for individual organs, usually for the two or three organs receiving the highest dose, and the EDE (effective dose equivalent) or ED (equivalent dose), in the case of diagnostic studies, are used to evaluate the radiation dose expected to be received, and thus the maximum amount of activity that should be administered. Obviously in therapeutic situations, the evaluation is more important, as the radiation dose received is much higher. For many years the Medical Internal Radiation Dose (MIRD) method has been used for calculating diagnostic doses. The MIRD committee of the Society of Nuclear Medicine provides a general framework for the dosimetry of administered radiopharmaceuticals. 


\subsection{Internal dosimetry methods}

\subsubsection{Basic concepts}

A generic equation for the absorbed dose in an organ is:

$$
D=\frac{k \tilde{A} \sum_{i} n_{i} E_{i} \phi_{i}}{m}
$$

Where

$D=$ absorbed dose (rad or Gy);

$\tilde{A}=$ cumulated activity $\left({ }^{\mu} \mathrm{Ci}\right.$ h or $\left.\mathrm{MBq} \mathrm{s}\right)$;

$n_{i}=$ number of particles with energy $E_{i}$ emitted per nuclear transition;

$E_{l}=$ energy per particle $(\mathrm{MeV})$;

$f_{i}=$ fraction of energy absorbed in the target;

$m=$ mass of target region ( $\mathrm{g}$ or $\mathrm{kg}$ ) and

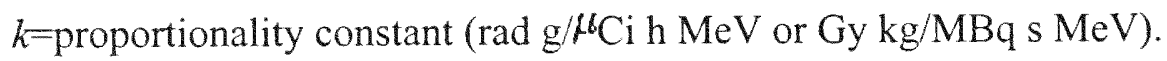

The term "cumulated activity" $(\tilde{A})$ is given to the area under the time-activity curve for a source organ or region. As activity is the number of disintegrations per unit time, integrating this over time gives the total number of disintegrations.

\subsubsection{The MIRD Formulation}

The Medical Internal Radiation Dose (MIRD) Committee of the Society of Nuclear Medicine has provided guidance on methods for calculating radiation absorbed dose estimates since 1968 (Watson and Stabin, 1993). By definition, the absorbed dose is the energy absorbed from ionizing radiation per unit mass of tissue. Because absorbed dose from internally distributed radionuclides is never completely uniform (Loevinger and 
Watson, 1988) the MIRD equations give the average, or mean, absorbed dose to a volume of tissue.

The schema is useful for estimating absorbed dose to volumes as small as a cluster of cells or as large as the total body. Microdosimetric techniques that account for statistical aspects of particle' track structures and energy distribution patterns in microscopic volumes can be used to express energy deposition in tissues from materials labeled with alpha-particle or Auger-electron emitters, particularly those incorporated within cells.

The equation for absorbed dose in the Medical Internal Radiation Dose (MIRD) system (Watson, 1988) is a deceptively simple representation of above equation:

$$
D=\tilde{A} S
$$

The cumulated activity is defined above, while all other terms are lumped in the factor $S$ :

$$
S=\frac{k \sum_{i} n_{i} E_{i} \phi_{i}}{m}
$$

In the MIRD equation, the factor $k$ traditionally applied is 2.13 , which gives absorbed dose in rad, from activity in ${ }^{\mu} \mathrm{Ci}$, mass in $\mathrm{g}$ and energy in $\mathrm{MeV}$. With more applications currently employing the SI unit system, a factor relating absorbed dose in Gy from activity in $\mathrm{Bq}$ and energy in $\mathrm{MeV}$ may be derived and employed. 
The total mean absorbed dose in a target region is calculated by summing the doses from all source regions to the target. Equation (1) can be divided into two types of parametersphysical and biological.

In any real internal dose problem, there will be more than one organ, which concentrates the activity, and many targets for which the absorbed dose is required. In this case, the MIRD equation needs to be solved for each source region $\left(r_{h}\right)$ and target region $\left(r_{k}\right)$ as follows:

$$
D_{r_{k}}=\sum_{h} \tilde{A}_{h} S\left(r_{k}-r_{h}\right)
$$

If the area under the time-activity curve for a source organ (cumulated activity) is normalized to the amount of activity administered $\left(A_{0}\right)$, this is defined as "residence time" (Watson, 1988).

$$
\mathrm{T}_{h}=\frac{\tilde{A}_{h}}{A_{0}}
$$

Using this definition the dose equation may be written as:

$$
D_{r}=A_{0} \sum_{h} T_{h} S\left(r_{k}-r_{h}\right)
$$

Values of cumulated activity $(\tilde{A})$ or residence time $(\tau)$ must be developed for those organs where the activity concentrates (e.g. liver, kidneys, spleen, thyroid), the organs involved in excretion of the compound from the body (e.g. urinary bladder, intestines) and the remainder of the body. Then, the absorbed dose is calculated by multiplying the values of $\tilde{\mathrm{A}}$ (or $\tau$ ) by the appropriate $\mathrm{S}$ values. The software package MABDOSE (Johnson, 1988) performs dose calculations, with the possible inclusion of tumor source 
regions, and integration of organ time-activity curves(the MIRDOSE software requires that the user perform these integrations separately).

\subsection{Absorbed dose calculation}

\subsubsection{MIRD $S$ values}

For homogeneous activity distribution in organs, MIRD $S$ values (Watson, 1988) have been used in both diagnostic and therapy absorbed dose calculations. Program packages (Johnson 1988) have been proposed; and the inclusion of tumors is possible. The largest single source of error in the procedure is often in the biokinetics, especially the uncertainty in the late activity-time data. However, in situations in which the mass of the target region is difficult to determine, this may introduce the largest source of error. The use of $S$ values based on standardized individuals, even if scaled using the true organ mass from patient-specific data, may also introduce significant errors into the analysis (Sgouros et. al., 1997). If the activity distribution is relatively uniform within the organ, the standard approximations may be relatively good, but if there are important in homogeneities (the presence of a hot or cold tumor, etc.), calculations based on the assumption of a uniform activity distribution may be significantly in error.

\subsection{Patient - Specific Dosimetry}

\subsubsection{Motivation for patient-specific dosimetry}

Radionuclide therapy based on patient-specific dosimetry offers the potential for optimizing the dose delivered to the target tumor through utilization of measured 
radiopharmaceutical kinetics specific to the individual. The administered activity may be tailored for the patient such that the highest possible radiation dose may be given to the tumor while limiting the dose to critical organs and tissues below any designated threshold for negative biological effects. A pretreatment quantitative dosimetry work-up using diagnostic ("tracer") activities of the therapy radiopharmaceutical serves also to identify those cancer patients for whom the treatment is likely to be most effective while eliminating those for whom it would be unsuccessful. In the case of radio immunotherapy, these considerations are of particular importance in that the low uptake in tumor regions (low target to non-target uptake ratios) may constrain the treatment protocol (Erdi and Wessels 1996).

\subsubsection{Activity Quantitation (Introduction to SPECT/CT):}

With SPECT systems, simultaneous transmission-emission studies can be made with exact registration, but the spatial resolution characteristic of SPECT allows visualization only of major features such as organ outlines and lung regions. In many patient situations, a conventional CT study is routinely made prior to radionuclide therapy. Effective image registration techniques can be applied to take advantage of these anatomical images for the attenuation correction and for the 3D absorbed dose calculation. Potential problems include the fact that the CT data are obtained from X-ray spectra, thus the transformation of pixel values to attenuation coefficients and density values may not be straightforward.

Furthermore, the registration of SPECT/CT images is more complex in the abdomen, where translational and rotational effects may occur. 
Medical diagnoses commonly rely on assessment of a patient's functional status and physical condition, and there are superb imaging modalities capable of providing that information-within certain parameters. Radionuclide-tracer techniques such as positronemission tomography (PET) and single-photon-emission computed tomography (SPECT) provide functional information, but have relatively poor spatial resolution and can lack the anatomical information needed to localize or stage disease.

$\mathrm{CT}$, on the other hand, offers excellent spatial resolution and rich anatomical detail. Integrating the two on a common gantry and patient table, in a concept that has only recently been brought to the commercial marketplace, holds the promise of simplifying patient handling, data acquisition, and co registration of the CT and radionuclide image data. SPECT/CT and PET/CT operate on the same basic design principle: the dual modality acquires CT and radionuclide scans by translating the patient from one detector to the other while the patient remains on the table. This allows both images to be taken with consistent scanner geometry and with minimal delay between the two acquisitions. After both sets of images have been acquired and reconstructed, image-registration software fuses the images while accounting for differences in scanner geometry and image format between the two data sets. One of the major anticipated uses of SPECT/CT is the production of better attenuation correction. The results of both studies are available on the same day, so the pathology and the patient's medical condition obviously have not changed. Combined technology helps because it produces the result right away, with the appropriate technical subtleties. An additional benefit of SPECT/CT is its ability to 
quantitate blood flow in an absolute sense, which is important for the better detection of global balanced ischemia.

PET/CT also has the advantage of shortening overall examination time, thereby increasing throughput in the imaging center; a PET/CT fusion scanner can often image up to 16 cases per day.

\subsection{Absorbed Dose Calculation Methods for Patient Specific Dosimetry}

\subsubsection{Dose point kernels}

For infinite and homogeneous media, the dose point kernel (DPK) method can be used and also for an inhomogeneous activity distribution in a homogeneous material (regarding both elemental composition and density), a dose point kernel may be used (Berger, 1973; Prestwitch, 1989; Simpkin, 1990; Leichner, 1994). The DPK method could be applied for the situations involving finite and/or heterogeneous media by rescaling the DPKs, in spite of possible error in the dose estimation. Treatment planning systems, based on dose point kernels, had been reported by several authors (Sgouros, 1993; Giap, 1994; Akabani, 1997). The conversion of activity to absorbed dose can be regarded as a filtering method, either as a convolution process in the spatial domain or as a multiplication in the frequency domain. When applied in the spatial domain, rescaling of the kernel distribution can be done when crossing a boundary. Applying the kernel in the frequency domain is often implemented to speed up the calculations. 


\subsubsection{Monte Carlo simulation}

The major limitation with dose kernels is that they can only describe the distribution in a uniform infinite medium. This affects the accuracy in the calculation of the dose distribution at interfaces between different attenuating media (such as between lung tissue and muscle tissue). The most accurate method is therefore to fully model the interaction of photons and electrons from a patient-specific activity distribution and attenuation map using a Monte Carlo transport method (Raeside, 1976).

In a treatment planning system, based on quantitative SPECT and Monte Carlo, the emission of photons and electrons are simulated and their paths are followed, using probability functions to govern their loss of energy, deflection/scattering angles and other events, as they pass through the system. Performing transport of all emitted particles and their secondary particles can account for in homogeneities, both in activity, elemental composition, and density.

Public domain Monte Carlo packages, such as EGS4 (Simpkin, 1990), MCNP and ITS (Briesmeister, 1993), are available and may be implemented in radionuclide treatment planning. More dedicated treatment planning programs, based on Monte Carlo simulations, have been reported (Furhang, 1997). Such systems use quantitative planaror SPECT images to establish the biokinetics of the radiopharmaceutical and the activity distribution. Information about the size, shape and mass of the organs may be obtained from these data by, for example, segmentation methods. The information derived from 
these sequential images provides the activity as a function of time, which can, as discussed above, be used to calculate the absorbed dose rate as a function of time and location within the subject. The dose rate is then integrated to obtain the cumulative dose to individual organs, regions, etc. These data may be analyzed as an absorbed dose distribution, in terms of total absorbed dose to different regions, dose-volume histograms, etc., with the hope that such information will provide a more comprehensive evaluation of the therapy effect.

\subsubsection{S Voxel kernel method}

The main drawback of the Monte Carlo method is its extended computational time. A second problem with a Monte Carlo simulation is that the surface of the organs described in Monte Carlo code is usually required to be in analytical form; ex., sphere, cylinder, plane, or a volume composed by the intersection of those surfaces. Thus, it is difficult to use CT 3-D anatomy in any Mote Carlo calculation without assuming crude approximations. In an attempt to make computations both patient-specific and real-time, we have developed an alternative method based on a voxel source kernel ie. S Voxel kernel method. Average absorbed dose to each voxel in the 3-D patient volume was then estimated by convolving the S voxel kernel method with the 3-D matrix of organ uptakes. A uniform activity concentration in each organ was obtained by dividing cumulated activity by the total number of voxels in that organ. Next, the average dose in each organ was calculated by averaging the doses in all the voxels within the organ. Statistical 
information, such as standard deviation of the average dose in each organ as well as the dose-volume histogram, was also obtained.

Notice that, although uniform activity distribution within each organ was initially assumed in our calculation, this was not a limitation in the $\mathrm{S}$ voxel kernel method itself. If the no uniform distribution of radioactivity was known, it could be incorporated into the method by using a radioactivity concentration lookup table. Furthermore, if a tumor is visible in a patient $\mathrm{CT}$ scan, tumor volumes can be obtained by drawing a boundary around the lesion. Given this volume, the activity concentration within the tumor can be determined using the CAMI (CT -assisted matrix inversion method) bed dose to tumor can be estimated by the S voxel kernel method. 


\section{MATERIALS AND METHODS}

\subsection{Calculation of Svoxel Values:}

Voxel $\mathrm{S}$ values could be calculated from the six-dimensional numerical integration of dose-point kernels over the source and target voxels or by the convolution of dose pointkernels with the geometrical factor of the source-target voxel geometry. In this research S values are calculated, based on the Monte Carlo volume integration of tabulated dose point-kernels of beta emissions over a voxel-to- voxel geometry.

The method has been implemented in $\mathrm{C}++$ Program (Windows NT) and this method calculates beta $\mathrm{S}$ values from the dose point-kernels tabulated by Cross (Cross et. al., 1992) and any cubical or non-cubical voxel dimensions in a water medium. Results of calculations were verified by comparing voxel $\mathrm{S}$ values of four radionuclides and three voxel sizes with those reported by Bolch (Bolch et. al., 1999) using the EGS4 code for Monte Carlo radiation transport simulation.

Beta dose point-kernels in water were derived from the tables of Cross (Cross et. al., 1992) are stored as a lookup table for each radionuclide. The units used for dose rate in Cross are $\mathrm{nGy} / \mathrm{Bq}-\mathrm{h}$, so dose point-kernel data of Cross were multiplied by 0.0002778 to convert the dose rate from $\mathrm{nGy} / \mathrm{Bq}-\mathrm{h}$ to $\mathrm{mGy} / \mathrm{MBq}-\mathrm{s}$. 
First column represents distance in $\mathrm{mm}$ and second column in the table represents dosepoint kernels in nGy/Bq-h.

\begin{tabular}{|l|}
\hline data: 29 \\
$0.000,104.0$ \\
$0.008,99.60$ \\
$0.016,99.20$ \\
$0.024,98.90$ \\
$0.032,98.60$ \\
$0.040,98.30$ \\
$0.080,96.40$ \\
$0.120,93.50$ \\
$0.160,89.70$ \\
$0.200,85.10$ \\
$0.240,79.60$ \\
$0.280,73.60$ \\
$0.320,67.10$ \\
$0.360,60.40$ \\
$0.400,53.50$ \\
$0.440,46.60$ \\
$0.480,39.70$
\end{tabular}


3.2 Target and Source voxels: The centroid of the source voxel was considered as $(0,0,0)$ and the centroid of the target voxel as $(x, y, z)$ where these values are the distance in $\mathrm{mm}$ between the centroids of the source and target voxels for each axis.

3.3 Random points in Source and Target voxel: For each pair of source and target voxels, a number of rays were simulated between random points $\left(\mathrm{X}_{\mathrm{s}, \mathrm{Ys}} \mathrm{Zs}\right)$ within the source voxel and random points $\left(\mathrm{x}_{\mathrm{t}}, \mathrm{Y}_{\mathrm{t}}, \mathrm{Zt}\right)$ within the target voxel. Random points within the source and target voxels were determined by using Random Numbers as:

$$
\begin{aligned}
& X s=(\Delta x / 2) \cdot R N D(1) \\
& Y s=(\Delta y / 2) \cdot R N D(2) \\
& Z s=(\Delta z / 2) \cdot \operatorname{RND}(3), \\
& X t=x+(\Delta x / 2) \cdot R N D(4) \\
& Y t=y+(\Delta y / 2) \cdot R N D(5) \\
& Z t=z+(\Delta z / 2) \cdot R N D(6),
\end{aligned}
$$

where $\Delta x, \Delta y$ and $\Delta z$ are the edge dimensions of the cubical or non-cubical voxel, and $\mathrm{RND}\left({ }^{*}\right)$ is a random number uniformly distributed between -1 and +1 . 


\subsection{Explanation of Random number generations Program:}

The function rand ( ) generates random numbers from 0 to 65534 , where 65534 is the maximum random number. But as a part of this research work, the generation of random numbers from -1 to +1 is necessitated. Hence the modified program given above, meets the specified needs. In this program, first the random values from 0 to 65534 have been generated and then checked for a condition, i.e., if the random value is greater than 1 then it is divided by RAND_MAX. By doing this we can get random numbers from 0 to 1 . And now for generating random numbers from -1 to 0 , we generate two random numbers randNum 1 and randNum2 and one integer randPow. Where

randPow $=$ randNum $* 10$

Now this randPow gives only single digit random numbers (ex, $3,7,6,9,2,1 \ldots)$ as this integer is multiplied by 10 .

randNum $1=$ pow $(-1$, randPow $){ }^{*}$ randNum2

pow(-1,randPow) generates the random signs (+ve or -ve)

Sign is assigned to random numbers generated by randNum 2 variable by multiplying with pow $(-1$,randPow $)$ and are stored in randNum 1 .

\subsection{Diagram to check Random Numbers between -1 and +1 :}

The random numbers generated were plotted in excel graph and the mean and standard deviation were calculated. The mean value is zero and we can say that the random numbers generated were in between -1 and +1 . And the standard deviation is 0.5 . 
Diagram to check the random numbers between -1 and +1

randomnumbers

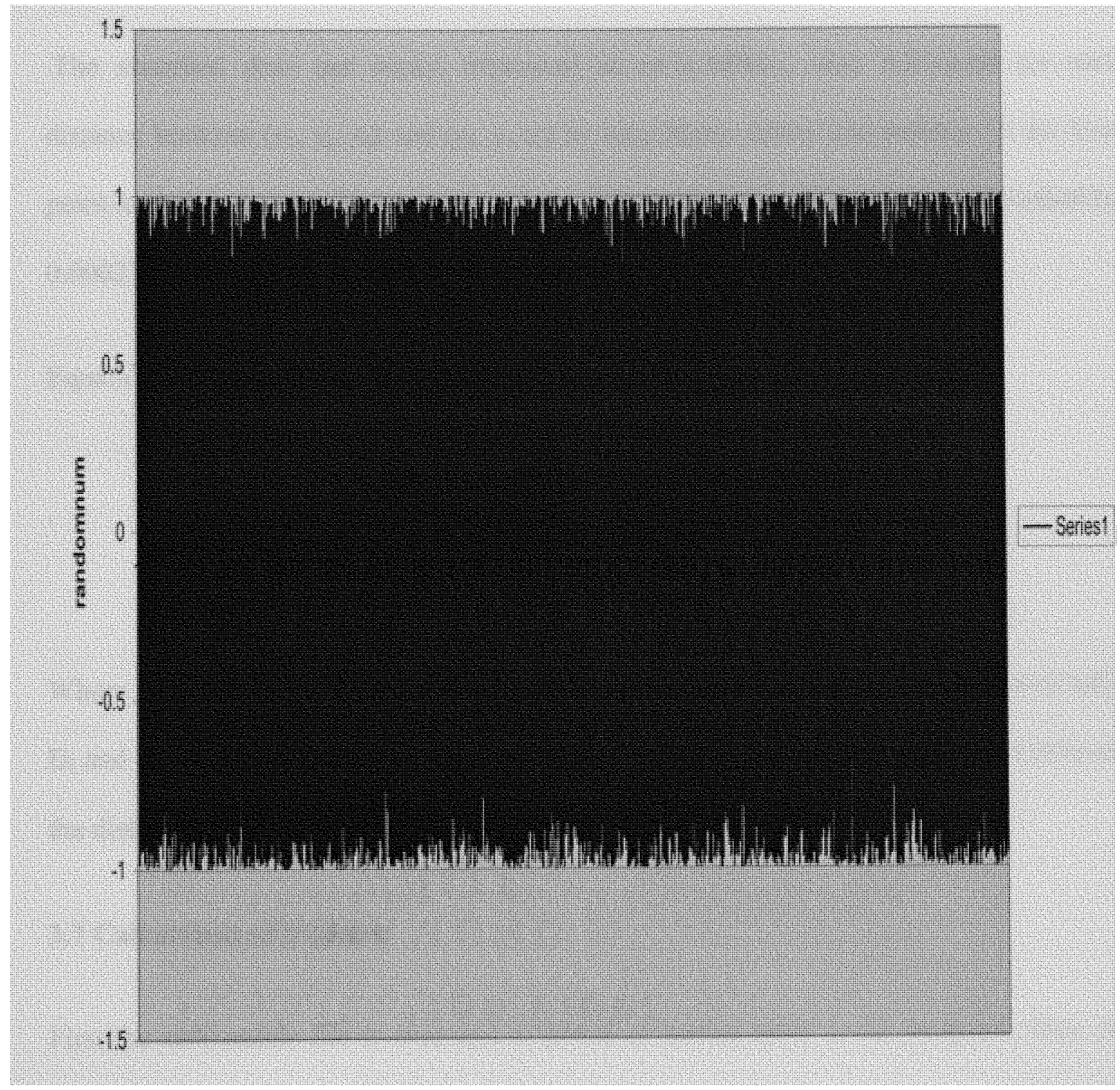




\subsection{Linear logarithmic Interpolation:}

At the position $(0,0,0)$ the target voxel was the same as source voxel. No dose calculation was performed if source and target random points become the same at voxel $(0,0,0)$.

The absorbed dose per decay, expressed in $\mathrm{mGy} / \mathrm{MBq}-\mathrm{s}$, was calculated for each distance between $\left(x_{s}, y_{s}, z_{s}\right)$ and $\left(x_{t}, y_{t}, z_{t}\right)$ by logarithmic-linear interpolation of the dose point-kernel data in the lookup table. Dose point-kernel data of Corss were multiplied by 0.0002778 to convert dose rate from $\mathrm{nGy} / \mathrm{MBq}-\mathrm{s}$.

Equation for Logarithmic Interpolation :

$$
\frac{\log [\mathrm{r}(\mathrm{i})]-\log [\mathrm{r}(\mathrm{i}+1)]}{\log [\mathrm{r}(\mathrm{i})]-\log [\mathrm{Y}]}=\frac{\left(\mathrm{d}_{\mathrm{i}}-\mathrm{d}_{\mathrm{i}+1}\right)}{\left(\mathrm{d}_{\mathrm{i}}-\mathrm{X}\right)}
$$

Where $\mathrm{r}(\mathrm{i})$ and $\mathrm{r}(\mathrm{i}+1)$ are the values of dose rate in $\mathrm{nGy} / \mathrm{Bq}-\mathrm{h}$ derived from Bolch table $D_{i}$ and $d_{i+1}$ are the distances in $\mathrm{cm}$ from Bolch table $\mathrm{X}$ is the distance which lies between the distances in the table $\mathrm{Y}$ is the value of dose rate at distance $\mathrm{X}$.

\subsection{Calculation of Svalues:}

First the radionulide file is read and is stored as lookup table in the program. Then the voxel file is also read, which has pixel dimensions and also maximum values of the centroids of target voxels in positive octant and also the number of simulations. Depending on the centroids and no. of simulations the voxel $\mathrm{S}$ values are calculated from the lookup table. Voxel S values were calculated as the average of all absorbed doses per 
decay. One million rays were simulated for each source-target voxel pair. After one million of simulations, there was no reduction in the relative standard deviation (STD/average) of voxel S values. Results of one million or more simulations were also closer to those of Bolch (Bolch et. al., 1999). Ten million rays were simulated in the case of low energy emissions to improve the statistical uncertainty of the dose. Since the radial symmetry of dose point-kernel, voxel $\mathrm{S}$ values were only calculated in the positive octant. The voxel Svalues lower than $0.01 \%$ of the voxel $S$ values at $(0,0,0)$ were discarded. The average of one million Svalues is calculated for all distances and for all coordinates.

The distance between the source and target random points is calculated by :

$$
D=\sqrt{ }\left(X_{t}-X_{s}\right)+\left(Y_{t}-Y_{s}\right)+\left(Z_{t}-Z_{s}\right)
$$

The distances, which are very less, are neglected. When the distance lies between the values in the Bolch table then the dose point kernel for that distance is calculated by using Interpolation method. The formula for Interpolation is given as:

$$
\begin{gathered}
Y_{x}=(\log [r(i)]-\log [r(i+1)]) *\left(d_{i}-X\right) /\left(d_{i}-d_{i+1}\right) \\
S_{x}{ }^{1}=\exp \left(\log [r(i)]-Y_{x}\right) \\
\text { Svoxel }=S_{x}{ }^{1} / D * D \\
\text { Svoxel }=\text { Svoxel } * 0.0002778 \\
\text { Stotal = Stotal }+ \text { Svoxel }
\end{gathered}
$$


Where $r(i)$ and $r(i+1)$ are the values of dose rate in $n G y / B q-h$ derived from Bolch table

$\mathrm{X}$ is the distance, which we need

$Y_{x}$ is the value of dose rate at distance $\mathrm{X}$.

Svoxel is the absorbed dose calculated from Logarithmic Interpolation

Stotal is the total of all absorbed doses for one million simulations

Saverage is the average of all absorbed doses.

\subsection{Flowchart of Complete Program}

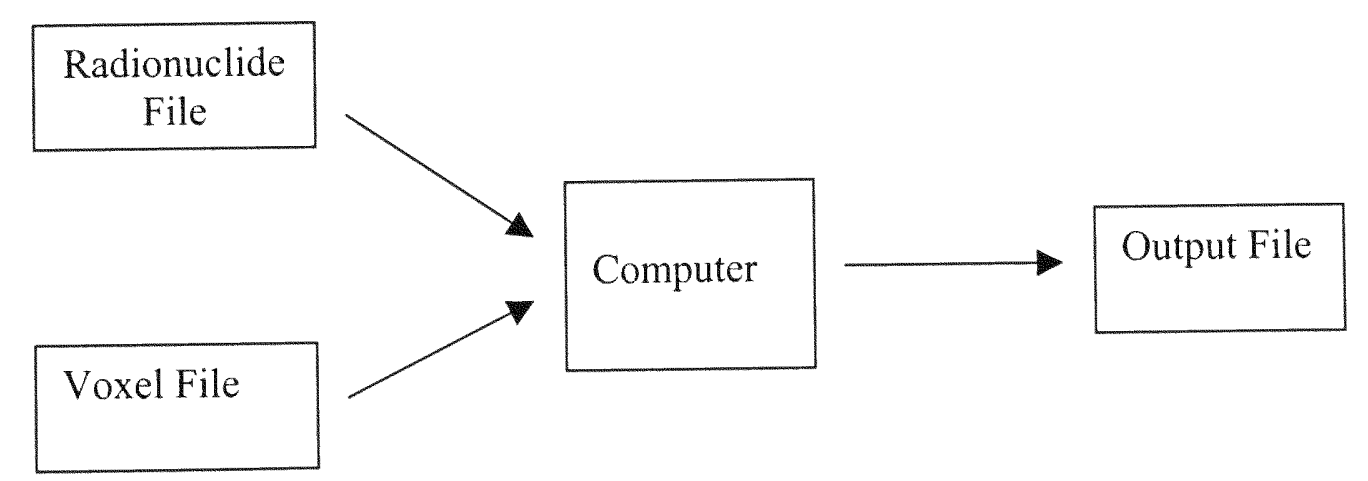

\subsection{Inputs in program}

To run the program the user must input the radionuclide, voxel dimension and the number of simulations. 
There are two input files in this program: 1) Radionuclide file 2) Voxel file

\section{Radionuclide File: Y90}

Radionuclide file has beta dose-point kernels in water for different distances which are derived from the tables of Cross (Cross et al., 1992).

It had two columns, the first represents the distance in $\mathrm{mm}$ and the second one represents dose-point kernel values in $\mathrm{nGy} / \mathrm{Bq}-\mathrm{h}$

$$
\begin{aligned}
& \text { data: } 29 \\
& 0.000,104.0 \\
& 0.008,99.60 \\
& 0.016,99.20 \\
& 0.024,98.90 \\
& 0.032,98.60 \\
& 0.040,98.30 \\
& 0.080,96.40 \\
& 0.120,93.50 \\
& 0.160,89.70
\end{aligned}
$$

\section{Voxel File}

The other input file ie. the voxel file has pixel dimensions and also maximum values of the centroids of target voxels in positive octant and number of simulations. 
The voxel sizes are in $\mathrm{cm}$.

xpixel: 0.3

ypixel: 0.3

zpixel: 0.3

simulations: 10000000

Max xposition: 6

Max yposition: 6

Max zposition: 6 


\subsubsection{Program Output}

The program output is a text file. It includes voxel $\mathrm{S}$ values for the set of coordinates in the first octant.

\begin{tabular}{l} 
Output File: \\
Radionuclide: Y90.dat \\
X: 3 \\
Y: 3 \\
Z: 3 \\
Xsize: 0.300000 \\
Ysize: 0.300000 \\
Zsize: 0.300000 \\
000: 1.612986 \\
100: 0.282232 \\
200: 0.021710 \\
\hline
\end{tabular}

This program can be used to calculate voxel $\mathrm{S}$ values of beta emissions from tabulated dose-point kernels and for any combination of pixel edges and thickness of SPECT and PET images without the complexity and expertise needed for direct Monte Carlo radiation transport simulations. Total time of calculation is from 5 to 60 minutes, depending on voxel dimensions, no. of simulations and radionuclide. 


\subsection{FlowChart.}

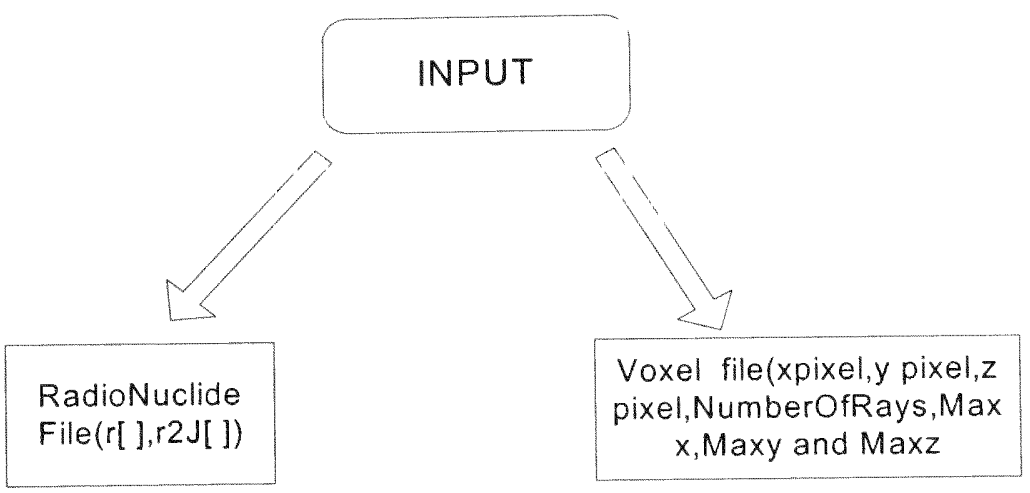

$\mathrm{r} 2 \mathrm{~J}[\mathrm{]}=\mathrm{r} 2 \mathrm{~J}[\mathrm{]}$ * 0.0002778
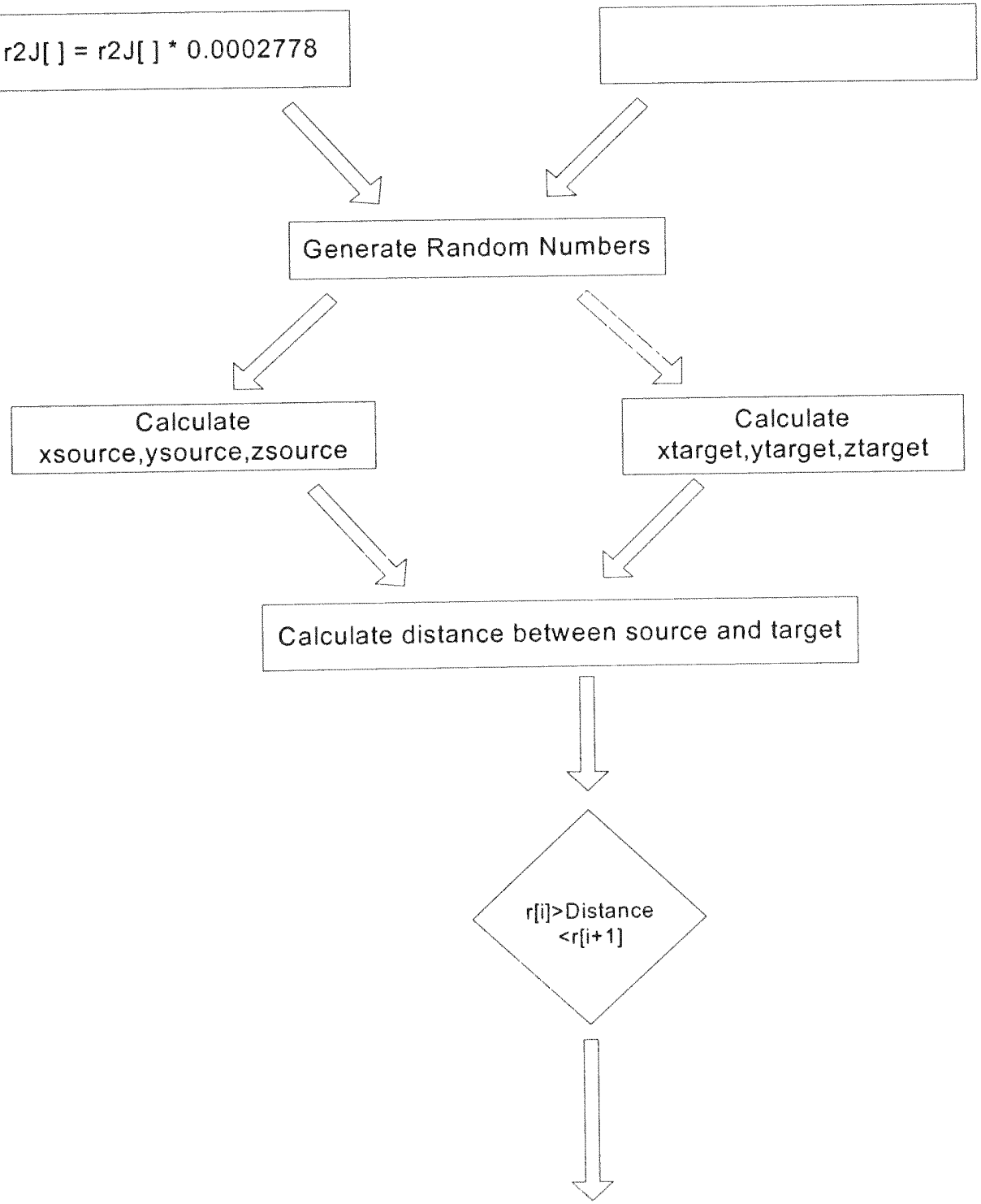
Calculate $S$ value using logarithmic interpolation

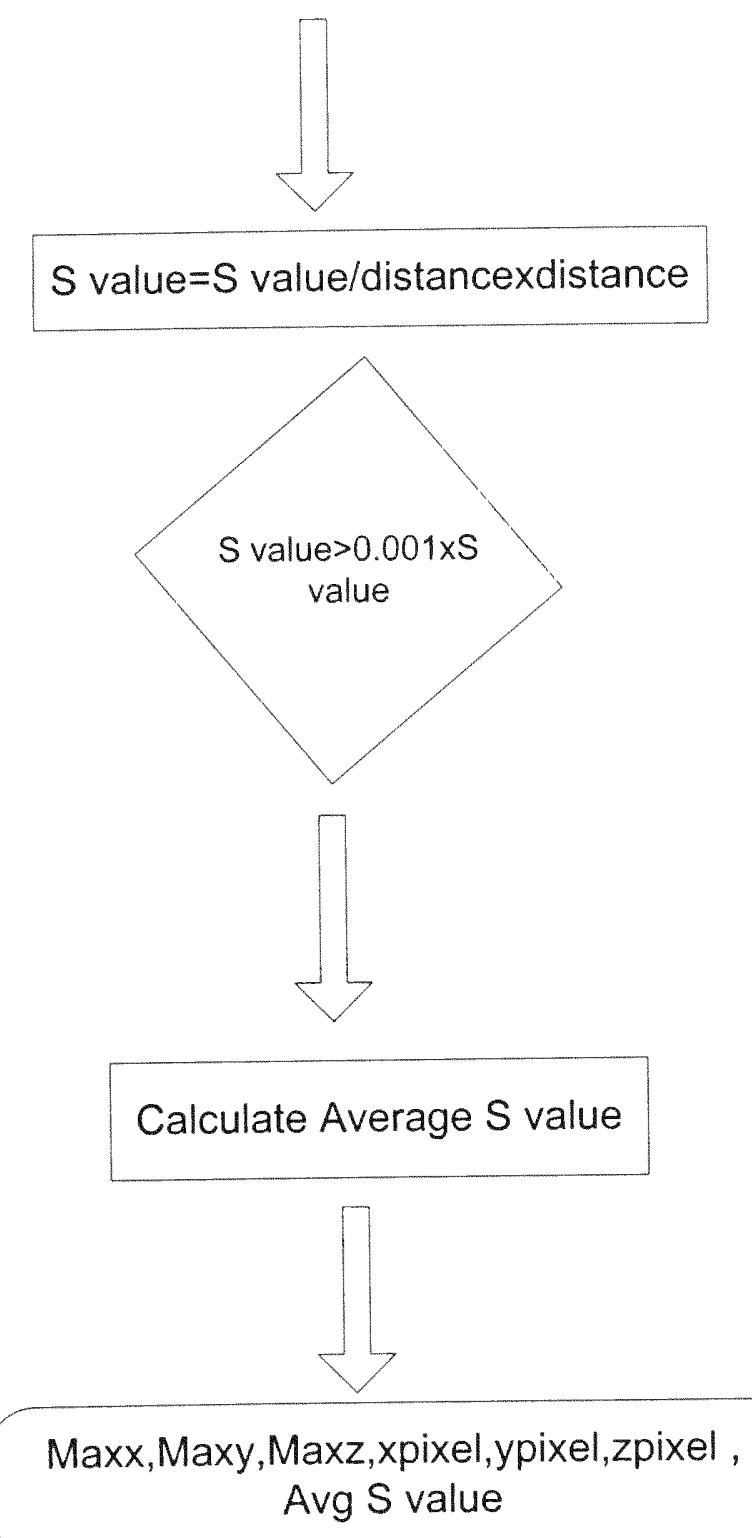

And these values are compared with that of the Bolch Method by calculating the percentage difference in both methods. 


\section{RESULTS}

Isotopes: Cu-64, Cu-67, I-125, I-131, In-111, P-32, Re-186, Re-188, Sr-89, Y-90 Pixel Size: $1 \mathrm{~mm}$

Svalues in $\mathrm{mGy} / \mathrm{MBq}-\mathrm{s}$.

\begin{tabular}{|c|c|c|c|c|c|c|}
\hline$x y z$ & $\begin{array}{l}\text { distance } \\
(\mathrm{mm})\end{array}$ & $\mathrm{Cu}-64$ & $\mathrm{Cu}-67$ & $\mid-125$ & $\mid-131$ & $\ln -111$ \\
\hline 000 & 0.00 & 11.009109 & 16.6778 & 0.650181 & 16.9651 & 3.159351 \\
\hline 001 & 1.00 & 1.037525 & 0.97515 & & 1.521666 & 0.168921 \\
\hline 011 & 1.41 & 0.191359 & 0.112997 & & 0.259719 & 0.010566 \\
\hline 111 & 1.73 & 0.045394 & 0.017664 & & 0.05748 & 0.000738 \\
\hline 002 & 2.00 & 0.007272 & & & 0.007974 & \\
\hline 012 & 2.24 & 0.002089 & & & 0.002182 & \\
\hline 211 & 2.45 & & & & & \\
\hline 220 & 2.83 & & & & & \\
\hline 221 & 3.00 & & & & & \\
\hline 222 & 3.16 & & & & & \\
\hline 300 & 3.00 & & & & & \\
\hline 310 & 3.16 & & & & & \\
\hline 311 & 3.32 & & & & & \\
\hline
\end{tabular}

\begin{tabular}{|c|c|c|c|c|c|c|}
\hline \multirow[b]{2}{*}{$x y z$} & \multicolumn{6}{|c|}{ distance } \\
\hline & $(\mathrm{mm})$ & P-32 & $\operatorname{Re}-186$ & $\operatorname{Re}-188$ & Sr-89 & $Y-90$ \\
\hline 0 & 0.00 & 4.023276 & 19.73106 & 16.98634 & 16.71453 & 15.35531 \\
\hline 100 & 1.00 & 0.706466 & 2.654223 & 3.223802 & 3.17959 & 3.173595 \\
\hline 110 & 1.41 & 0.247988 & 0.808911 & 1.41814 & 1.333232 & 1.444692 \\
\hline 111 & 1.73 & 0.113397 & 0.342775 & 0.852372 & 0.76223 & 0.889774 \\
\hline 200 & 2.00 & 0.055137 & 0.156632 & 0.577127 & 0.489365 & 0.618016 \\
\hline 210 & 2.24 & 0.030619 & 0.085181 & 0.433226 & 0.350555 & 0.47482 \\
\hline 211 & 2.45 & 0.017331 & 0.04775 & 0.338301 & 0.26048 & 0.379715 \\
\hline 220 & 2.83 & 0.005578 & 0.015489 & 0.222442 & 0.153994 & 0.262015 \\
\hline 221 & 3.00 & 0.00318 & 0.009051 & 0.185499 & 0.121319 & 0.223791 \\
\hline 222 & 3.16 & 0.00054 & & 0.114786 & 0.062347 & 0.148844 \\
\hline 300 & 3.00 & 0.003019 & 0.0086 & 0.184792 & 0.120718 & 0.223139 \\
\hline 310 & 3.16 & 0.001705 & 0.005043 & 0.156171 & 0.096166 & 0.193143 \\
\hline 311 & 3.32 & 0.000948 & 0.002946 & 0.133267 & 0.077166 & 0.16879 \\
\hline
\end{tabular}

Table I. Voxel S values (mGy/MBq.s) of cubical voxels of $1 \mathrm{~mm}$ on edge. 
Isotopes: Cu-64, Cu-67, I-125, I-131, In-111 P-32, Re-186, Re-188, Sr-89, Y-90

Pixel Size: $2 \mathrm{~mm}$

Svalues in $\mathrm{mGy} / \mathrm{MBq}-\mathrm{s}$.

\begin{tabular}{|c|c|c|c|c|c|c|}
\hline$x y z$ & $\begin{array}{c}\text { distance } \\
\text { (mm) }\end{array}$ & $\mathrm{Cu}-64$ & $\mathrm{Cu}-67$ & |-125 & $\mid-131$ & $\ln -111$ \\
\hline 000 & 0.00 & 1.721667 & 2.654138 & 0.128429 & 2.724808 & 0.457736 \\
\hline 001 & 2.00 & 0.092957 & 0.074754 & & 0.133991 & \\
\hline 011 & 2.83 & 0.007769 & 0.004088 & & 0.010257 & \\
\hline 111 & 3.47 & 0.000782 & 0.00027 & & 0.000952 & \\
\hline 002 & 4.00 & & & & & \\
\hline 012 & 4.47 & & & & & \\
\hline 211 & 4.90 & & & & & \\
\hline 220 & 5.66 & & & & & \\
\hline 221 & 6.00 & & & & & \\
\hline 222 & 6.33 & & & & & \\
\hline 300 & 6.00 & & & & & \\
\hline 310 & 6.33 & & & & & \\
\hline 311 & 6.63 & & & & & \\
\hline
\end{tabular}

\begin{tabular}{|c|c|c|c|c|c|c|}
\hline$x y z$ & $\begin{array}{l}\text { listance } \\
(\mathrm{mm})\end{array}$ & P-32 & $\mathrm{Re}-186$ & Re-188 & Sr-89 & $Y=90$ \\
\hline 0 & 0.00 & 4.00025 & 3.790772 & 4.007126 & 3.893965 & 3.76913 \\
\hline 100 & 2.00 & 0.70643 & 0.336128 & 0.694345 & 0.618123 & 0.726269 \\
\hline 110 & 2.83 & 0.248216 & 0.056258 & 0.262648 & 0.199626 & 0.298646 \\
\hline 111 & 3.47 & 0.113491 & 0.011954 & 0.13309 & 0.083915 & 0.165006 \\
\hline 200 & 4.00 & 0.055152 & 0.001388 & 0.074108 & 0.036309 & 0.101215 \\
\hline 210 & 4.47 & 0.030624 & & 0.04666 & 0.018156 & 0.069196 \\
\hline 211 & 4.90 & 0.01734 & & 0.030303 & 0.009127 & 0.048883 \\
\hline 220 & 5.66 & 0.005574 & & 0.013447 & 0.002172 & 0.025713 \\
\hline 221 & 6.00 & 0.003178 & & 0.009133 & 0.001049 & 0.019 \\
\hline 222 & 6.33 & 0.000539 & & 0.002892 & & 0.007781 \\
\hline 300 & 6.00 & 0.003019 & & 0.008985 & 0.000919 & 0.01883 \\
\hline 310 & 6.33 & 0.001705 & & 0.006132 & 0.000428 & 0.013998 \\
\hline 311 & 6.63 & 0.000949 & & 0.004198 & & 0.010421 \\
\hline
\end{tabular}

Table II. Voxel S values (mGy/MBq.s) of cubical voxels of $2 \mathrm{~mm}$ on edge. 
Isotopes: $\mathrm{Cu}-64, \mathrm{Cu}-67, \mathrm{I}-125, \mathrm{I}-131, \mathrm{In}-111, \mathrm{P}-32, \mathrm{Re}-186, \mathrm{Re}-188, \mathrm{Sr}-89, \mathrm{Y}-90$

Pixel Size: $3 \mathrm{~mm}$

Svalues in $\mathrm{mGy} / \mathrm{MBq}-\mathrm{s}$.

\begin{tabular}{|c|c|c|c|c|c|c|}
\hline$x y z$ & $\begin{array}{c}\text { distance } \\
(\mathrm{mm})\end{array}$ & Cu-64 & $\mathrm{Cu}-67$ & $\mid-125$ & $\mid-131$ & $\ln -111$ \\
\hline 000 & 0.00 & 0.590493 & 0.772997 & 0.045001 & 0.911906 & 0.142114 \\
\hline 001 & 3.00 & 0.020665 & & & 0.02909 & \\
\hline 011 & 4.24 & 0.001123 & & & 0.001436 & \\
\hline 111 & 5.20 & 0.000067 & & & & \\
\hline 002 & 6.00 & & & & & \\
\hline 012 & 6.71 & & & & & \\
\hline 112 & 7.35 & & & & & \\
\hline 022 & 8.49 & & & & & \\
\hline 003 & 9.00 & & & & & \\
\hline 013 & 9.49 & & & & & \\
\hline 113 & 9.95 & & & & & \\
\hline 221 & 9.00 & & & & & \\
\hline
\end{tabular}

\begin{tabular}{|c|c|c|c|c|c|c|}
\hline$x y z$ & $\begin{array}{c}\text { distanc } \\
(\mathrm{mm})\end{array}$ & P.32 & $\operatorname{Re}-186$ & $\operatorname{Re}-188$ & Sr-89 & $Y=90$ \\
\hline 000 & 0.00 & 1.660587 & 1.32593 & 1.652983 & 1.618985 & 1.612986 \\
\hline 001 & 3.00 & 0.239203 & 0.082815 & 0.250747 & 0.197784 & 0.282232 \\
\hline 011 & 4.24 & 0.061359 & 0.008766 & 0.07571 & 0.044614 & 0.098632 \\
\hline 111 & 5.20 & 0.019515 & 0.001111 & 0.029632 & 0.01223 & 0.045009 \\
\hline 002 & 6.00 & 0.005448 & & 0.011737 & 0.002472 & 0.02171 \\
\hline 012 & 6.71 & 0.00199 & & 0.005556 & 0.000739 & 0.011849 \\
\hline 112 & 7.35 & 0.000715 & & 0.002644 & 0.000214 & 0.006514 \\
\hline 022 & 8.49 & & & 0.000563 & & 0.001913 \\
\hline 003 & 9.00 & & & 0.00026 & & 0.000957 \\
\hline 013 & 9.49 & & & 0.000217 & & 0.000501 \\
\hline 113 & 9.95 & & & & & 0.000255 \\
\hline 221 & 9.00 & & & & & 0.001028 \\
\hline
\end{tabular}

Table III. Voxel S values (mGy/MBq.s) of cubical voxels of $3 \mathrm{~mm}$ on edge. 
Isotopes: $\mathrm{Cu}-64, \mathrm{Cu}-67, \mathrm{I}-125, \mathrm{In}-111, \mathrm{P}-32, \mathrm{Re}-186, \mathrm{Re}-188, \mathrm{Sr}-89, \mathrm{Y}-90$

Pixel Size: $4 \mathrm{~mm}$

Svalues in $\mathrm{mGy} / \mathrm{MBq}-\mathrm{s}$.

\begin{tabular}{|c|c|c|c|c|c|}
\hline$x y z$ & $\begin{array}{c}\text { distance } \\
(\mathrm{mm})\end{array}$ & $\mathrm{Cu}-64$ & $\mathrm{Cu}-67$ & $\mid-125$ & In-111 \\
\hline 000 & 0.00 & 11.009109 & 0.000313 & 0.128429 & 0.000675 \\
\hline 001 & 4.00 & 1.037525 & & & \\
\hline 011 & 5.65 & 0.191359 & & & \\
\hline 111 & 6.93 & 0.045394 & & & \\
\hline 002 & 8.00 & 0.007272 & & & \\
\hline 012 & 8.95 & 0.002089 & & & \\
\hline 211 & 9.80 & & & & \\
\hline 220 & 11.32 & & & & \\
\hline 221 & 12.00 & & & & \\
\hline 222 & 12.65 & & & & \\
\hline 300 & 12.00 & & & & \\
\hline 310 & 12.65 & & & & \\
\hline 311 & 13.27 & & & & \\
\hline
\end{tabular}

\begin{tabular}{|ccccccc|}
\hline \multicolumn{2}{c}{ distance } & & & & & \\
xyz & $(\mathrm{mm})$ & P-32 & Re-186 & Re-188 & Sr-89 & Y-90 \\
\hline 0 & 0.00 & 0.001579 & 0.000718 & 0.001686 & 0.001285 & 0.00198 \\
100 & 4.00 & & & & & \\
110 & 5.65 & & & & & \\
111 & 6.93 & & & & & \\
200 & 8.00 & & & & & \\
210 & 8.95 & & & & & \\
211 & 9.80 & & & & & \\
220 & 11.32 & & & & & \\
221 & 12.00 & & & & & \\
222 & 12.65 & & & & & \\
300 & 12.00 & & & & & \\
310 & 12.65 & & & & & \\
311 & 13.27 & & & & & \\
\hline
\end{tabular}

Table IV. Voxel S values (mGy/MBq.s) of cubical voxels of $4 \mathrm{~mm}$ on edge. 
Isotopes: Cu-64, Cu-67, I-131, In-111, P-32, Re-186, Re-188, Sr-89, Y-90

Pixel Size: $5 \mathrm{~mm}$

Svalues in $\mathrm{mGy} / \mathrm{MBq}-\mathrm{s}$.

\begin{tabular}{|c|c|c|c|c|c|}
\hline$x y z$ & $\begin{array}{c}\text { distanc } \\
(\mathrm{mm})\end{array}$ & $\mathrm{Cu}-64$ & $\mathrm{Cu}-67$ & $\mid-131$ & $\ln -111$ \\
\hline 000 & 0.00 & 0.000127 & 0.0004 & 0.0003 & 0.000032 \\
\hline 001 & 5.00 & & & & \\
\hline 011 & 7.07 & & & & \\
\hline 111 & 8.67 & & & & \\
\hline 002 & 10.00 & & & & \\
\hline 012 & 11.18 & & & & \\
\hline 211 & 12.25 & & & & \\
\hline 220 & 14.15 & & & & \\
\hline 221 & 15.00 & & & & \\
\hline 222 & 15.82 & & & & \\
\hline 300 & 15.00 & & & & \\
\hline 310 & 15.82 & & & & \\
\hline 311 & 16.58 & & & & \\
\hline
\end{tabular}

\begin{tabular}{|c|c|c|c|c|c|c|}
\hline xyz & $\begin{array}{l}\text { istance } \\
(\mathrm{mm})\end{array}$ & P-32 & $\operatorname{Re}-186$ & $\operatorname{Re}-188$ & Sr-89 & Y-90 \\
\hline 0 & 0.00 & 0.000852 & 0.000339 & 0.000927 & 0.000654 & 0.001123 \\
\hline 100 & 5.00 & & & & & \\
\hline 110 & 7.07 & & & & & \\
\hline 111 & 8.67 & & & & & \\
\hline 200 & 10.00 & & & & & \\
\hline 210 & 11.18 & & & & & \\
\hline 211 & 12.25 & & & & & \\
\hline 220 & 14.15 & & & & & \\
\hline 221 & 15.00 & & & & & \\
\hline 222 & 15.82 & & & & & \\
\hline 300 & 15.00 & & & & & \\
\hline 310 & 15.82 & & & & & \\
\hline 311 & 16.58 & & & & & \\
\hline
\end{tabular}

Table V. Voxel S values (mGy/MBq.s) of cubical voxels of $5 \mathrm{~mm}$ on edge. 
Isotopes: Cu-64, Cu-67, I-131, In-111, P-32, Re-186, Re-188, Sr-89, Y-90

Pixel Size: $6 \mathrm{~mm}$

Svalues in $\mathrm{mGy} / \mathrm{MBq}$-s.

\begin{tabular}{|cccccc|}
\hline xyz & $\begin{array}{c}\text { distance } \\
(\mathrm{mm})\end{array}$ & Cu-64 & Cu-67 & l-131 & In-111 \\
\hline 000 & 0.00 & 0.00006 & 0.000061 & 0.000111 & 0.000027 \\
001 & 6.00 & & & & \\
011 & 8.48 & & & & \\
111 & 10.40 & & & & \\
002 & 12.00 & & & & \\
012 & 13.42 & & & & \\
211 & 14.70 & & & & \\
220 & 16.98 & & & & \\
221 & 18.00 & & & & \\
222 & 18.98 & & & & \\
300 & 18.00 & & & & \\
310 & 18.98 & & & & \\
311 & 19.90 & & & & \\
\hline
\end{tabular}

\begin{tabular}{|c|c|c|c|c|c|c|}
\hline$x y z$ & $\begin{array}{c}\text { distance } \\
(\mathrm{mm})\end{array}$ & P.32 & $\operatorname{Re}-186$ & $\mathrm{Re}-188$ & $\mathrm{Sr}-89$ & $Y-90$ \\
\hline 0 & 0.00 & 0.000459 & 0.000207 & 0.000546 & 0.000379 & 0.000635 \\
\hline 100 & 6.00 & & & & & \\
\hline 110 & 8.48 & & & & & \\
\hline 111 & 10.40 & & & & & \\
\hline 200 & 12.00 & & & & & \\
\hline 210 & 13.42 & & & & & \\
\hline 211 & 14.70 & & & & & \\
\hline 220 & 16.98 & & & & & \\
\hline 221 & 18.00 & & & & & \\
\hline 222 & 18.98 & & & & & \\
\hline 300 & 18.00 & & & & & \\
\hline 310 & 18.98 & & & & & \\
\hline 311 & 19.90 & & & & & \\
\hline
\end{tabular}

Table VI. Voxel S values (mGy/MBq.s) of cubical voxels of $6 \mathrm{~mm}$ on edge. 
Isotopes: $\mathrm{Cu}-64, \mathrm{Cu}-67, \mathrm{I}-125, \mathrm{I}-131, \mathrm{In}-111, \mathrm{P}-32$, Re-186, Re-188, Sr-89, Y-90

Pixel Size: $7 \mathrm{~mm}$

Svalues in $\mathrm{mGy} / \mathrm{MBq}-\mathrm{s}$.

\begin{tabular}{|c|c|c|c|c|c|c|}
\hline$x y z$ & $\begin{array}{c}\text { distance } \\
(\mathrm{mm})\end{array}$ & $\mathrm{Cu}-64$ & Cu-67 & $1-125$ & $\mid-131$ & In-111 \\
\hline 000 & 0.00 & 0.000041 & 0.00002 & 0.650181 & 0.000075 & 0.000012 \\
\hline 001 & 7.00 & & & & & \\
\hline 011 & 9.89 & & & & & \\
\hline 111 & 12.13 & & & & & \\
\hline 002 & 14.00 & & & & & \\
\hline 012 & 15.66 & & & & & \\
\hline 211 & 17.15 & & & & & \\
\hline 220 & 19.81 & & & & & \\
\hline 221 & 21.00 & & & & & \\
\hline 222 & 22.14 & & & & & \\
\hline 300 & 21.00 & & & & & \\
\hline 310 & 22.14 & & & & & \\
\hline 311 & 23.22 & & & & & \\
\hline
\end{tabular}

\begin{tabular}{|ccccccc|}
\hline distance & & & & & \\
(mm) & P-32 & Re-186 & Re-188 & Sr-89 & Y-90 \\
\hline 0 & 0.00 & 0.00029 & 0.000107 & 0.000322 & 0.000259 & 0.000384 \\
100 & 7.00 & & & & & \\
110 & 9.89 & & & & & \\
111 & 12.13 & & & & & \\
200 & 14.00 & & & & & \\
210 & 15.66 & & & & & \\
211 & 17.15 & & & & \\
220 & 19.81 & & & & \\
221 & 21.00 & & & & & \\
222 & 22.14 & & & & & \\
300 & 21.00 & & & & & \\
310 & 22.14 & & & & & \\
311 & 23.22 & & & & & \\
\hline
\end{tabular}

Table VII. Voxel $S$ values (mGy/MBq.s) of cubical voxels of $7 \mathrm{~mm}$ on edge. 
Distance is in $\mathrm{mm}$

Svalues are in $\mathrm{mGy} / \mathrm{Mbq}-\mathrm{s}$.

\begin{tabular}{|c|c|c|c|c|}
\hline Radionuclide & P32 & \multicolumn{3}{|c|}{} \\
\cline { 1 - 2 } Voxel Size & $3 \mathbf{m m}$ & \multicolumn{3}{|c|}{} \\
\hline Voxel & distance & Monte Carlo & Monte Carlo Radiation & \\
\hline Position & $\mathbf{m m}$ & Volume Integration & Transport Simulation & Diff (\%) \\
\hline 000 & 0 & 1.64 & 1.65 & 0.61 \\
\hline 001 & 3 & 0.239 & 0.232 & 2.89 \\
\hline 011 & 4.24 & 0.061 & 0.062 & 1.21 \\
\hline 111 & 5.2 & 0.0196 & 0.0212 & 7.74 \\
\hline 002 & 6 & 0.00545 & 0.00678 & 19.62 \\
\hline 012 & 6.71 & 0.00199 & 0.00288 & 30.94 \\
\hline 112 & 7.35 & 0.00072 & 0.00124 & 41.69 \\
\hline
\end{tabular}

Table VIII. Comparing Voxel S values of Radionuclide: P32 with Voxel size $3 \mathrm{~mm}$ using Monte Carlo Volume Integration (my method) and Monte Carlo Radiation Transport Simulation (Bolch Method) 
Distance is in $\mathrm{mm}$.

Svalues are in mGy/Mbq-s.

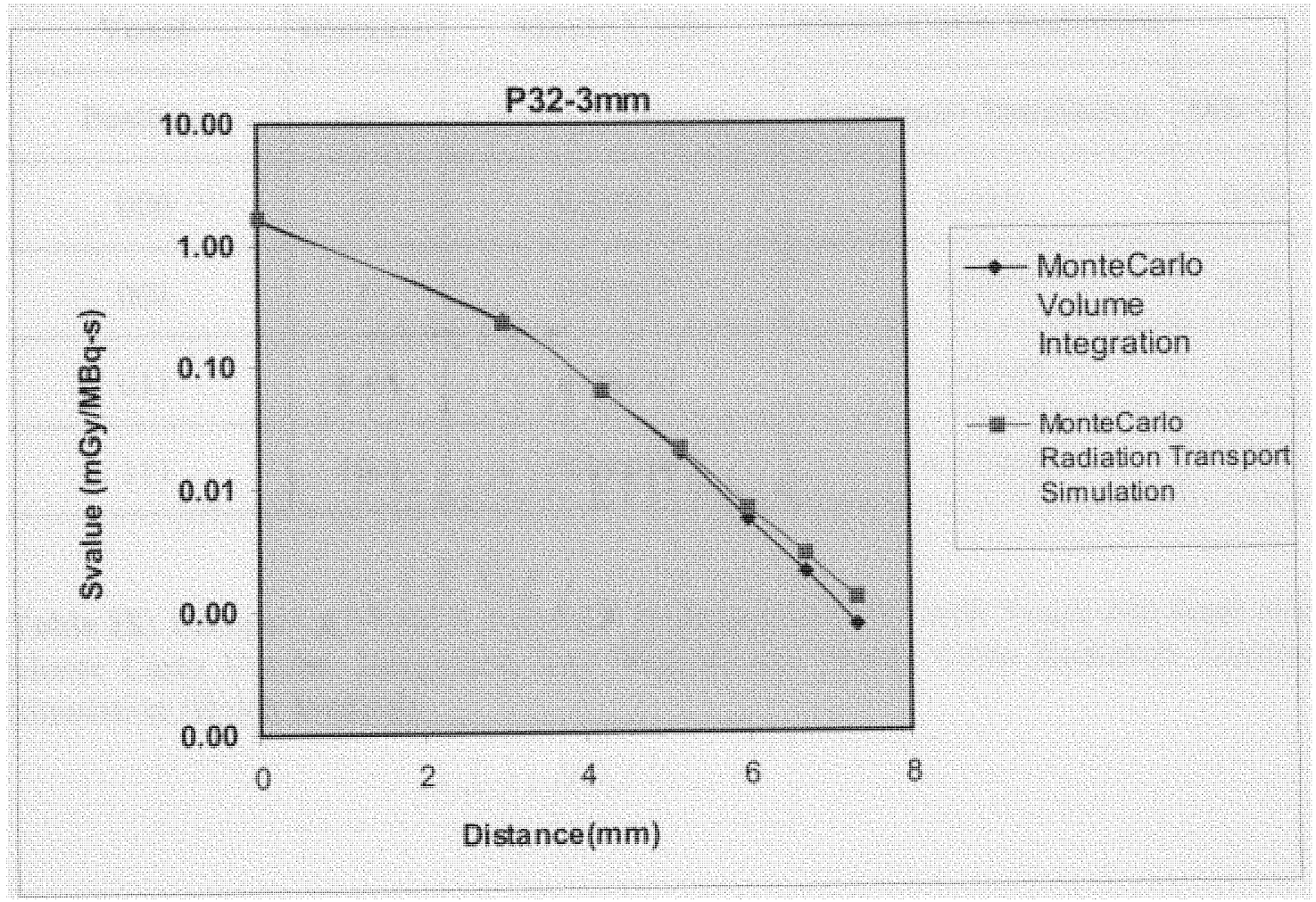

Figure 1: Comparing Voxel S values of Radionuclide: P32 with Voxel size $3 \mathrm{~mm}$ using Monte Carlo Volume Integration (my method) and Monte Carlo Radiation Transport Simulation (Bolch Method) 
Distance is in $\mathrm{mm}$.

Svalues are in $\mathrm{mGy} / \mathrm{Mbq}-\mathrm{s}$.

\begin{tabular}{|c|c|c|c|c|}
\hline Radionuclide & P32 & \multicolumn{3}{|c|}{} \\
\cline { 1 - 2 } Voxel Size & $6 \mathrm{~mm}$ & \multicolumn{3}{|c|}{} \\
\hline Voxel & Distance & Monte Carlo & Monte Carlo Radiation & \\
\hline Position & $\mathbf{m m}$ & Volume Integration & Transport Simulation & Diff (\%) \\
\hline 000 & 0 & 0.31913 & 0.319 & 0.04 \\
\hline 001 & 6 & 0.02512 & 0.0253 & 0.71 \\
\hline 011 & 8.48 & 0.00286 & 0.00313 & 8.53 \\
\hline 111 & 10.4 & 0.00038 & 0.00046 & 17.89 \\
\hline
\end{tabular}

Table IX: Comparing Voxel S values of Radionuclide: P32 with Voxel size $6 \mathrm{~mm}$ using Monte Carlo Volume Integration (my method) and Monte Carlo Radiation Transport Simulation (Bolch Method) 
Distance is in $\mathrm{mm}$.

Svalues are in $\mathrm{mGy} / \mathrm{Mbq}-\mathrm{s}$.

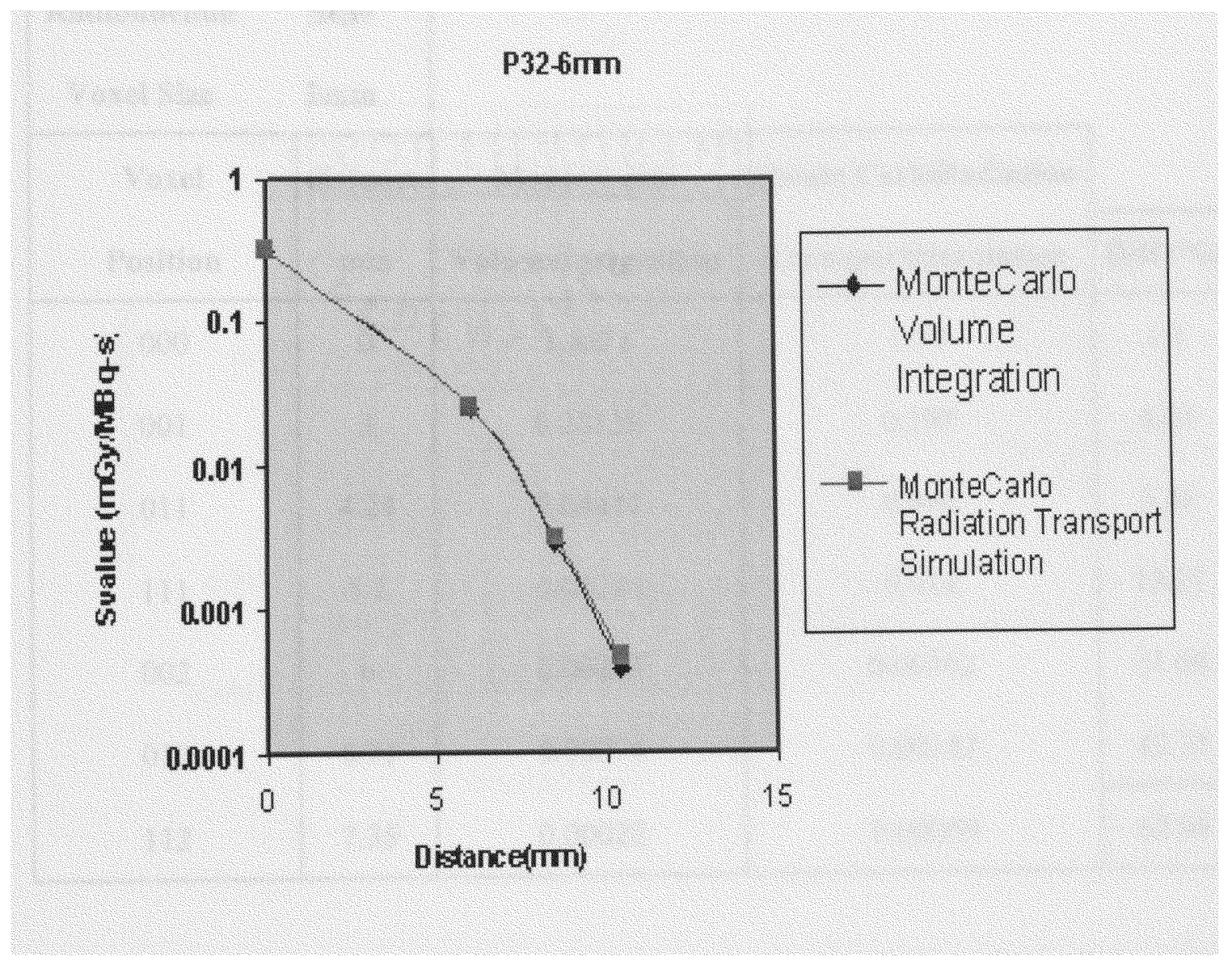

Figure 2: Comparing Voxel S values of Radionuclide: P32 with Voxel size $6 \mathrm{~mm}$ using Monte Carlo Volume Integration (my method) and Monte Carlo Radiation Transport Simulation (Bolch Method) 
Distance is in $\mathrm{mm}$.

Svalues are in $\mathrm{mGy} / \mathrm{Mbq}-\mathrm{s}$.

\begin{tabular}{|c|c|c|c|c|}
\hline $\begin{array}{l}\text { Radionuclide } \\
\text { Voxel Size }\end{array}$ & $\begin{array}{l}\text { Sr89 } \\
3 \mathrm{~mm}\end{array}$ & & & \\
\hline Voxel & distance & Monte Carlo & Monte CarloRadiation & \\
\hline Position & $\mathbf{m m}$ & VolumeIntegration & TransportSimulation & Diff $(\%)$ \\
\hline 000 & 0 & 1.5671 & 1.55 & 1.1 \\
\hline 001 & 3 & 0.20118 & 0.193 & 4.24 \\
\hline 011 & 4.24 & 0.04441 & 0.046 & 3.46 \\
\hline 111 & 5.2 & 0.01215 & 0.014 & 13.22 \\
\hline 002 & 6 & 0.00247 & 0.00362 & 31.66 \\
\hline 012 & 6.71 & 0.00074 & 0.00142 & 47.75 \\
\hline 112 & 7.35 & 0.00022 & 0.00059 & 63.08 \\
\hline
\end{tabular}

Table X. Comparing Voxel Svalues of Rdionuclide: $\mathrm{Sr} 89$ with voxel size $3 \mathrm{~mm}$ using Monte Carlo Volume Integration and Monte Carlo Radiation Transport Simulation method. 
Distance is in $\mathrm{mm}$.

Svalues are in $\mathrm{mGy} / \mathrm{Mbq}$-s.

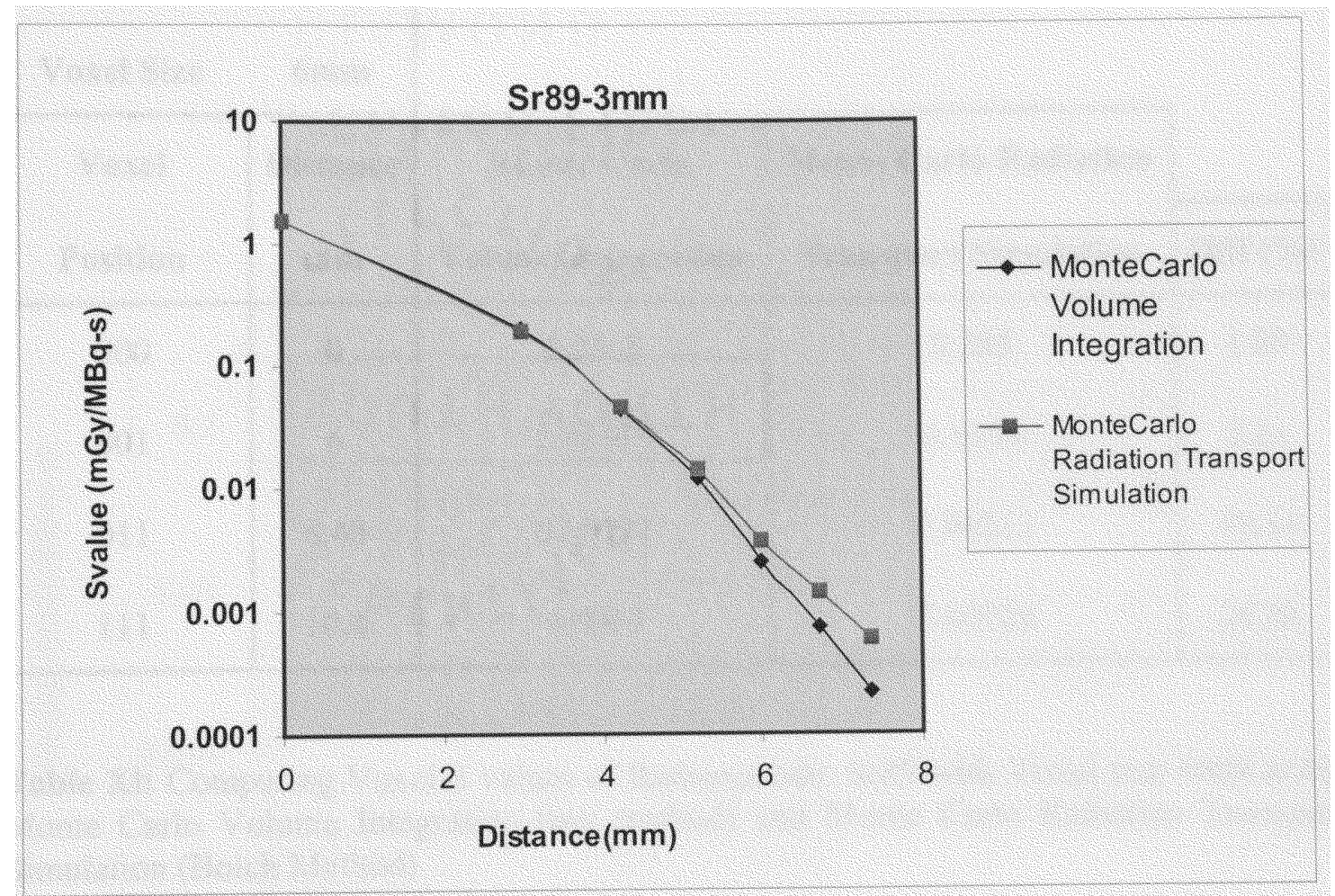

Figure 3: Comparing Voxel S values of Radionuclide: $\mathrm{Sr} 89$ with Voxel size $3 \mathrm{~mm}$ using Monte Carlo Volume Integration (my method) and Monte Carlo Radiation Transport Simulation (Bolch Method) 
Distance is in $\mathrm{mm}$.

Svalues are in $\mathrm{mGy} / \mathrm{Mbq}-\mathrm{s}$.

\begin{tabular}{|c|c|c|c|c|}
\hline \multicolumn{2}{|c|}{ Radionuclide } & \multicolumn{2}{|c}{ Sr89 } & \multicolumn{2}{|l|}{ Voxel Size } & $6 \mathrm{~mm}$ & \multicolumn{2}{|c|}{} \\
\hline Voxel & Distance & Monte Carlo & Monte Carlo Radiation & \\
\cline { 4 - 5 } Position & $\mathbf{m m}$ & Volume Integration & Transport Simulation & Diff (\%) \\
\hline 000 & 0 & 0.2815 & 0.285 & 1.23 \\
001 & 6 & 0.01917 & 0.0196 & 2.21 \\
011 & 8.48 & 0.00191 & 0.00213 & 10.14 \\
111 & 10.4 & 0.0002 & 0.00028 & 26.88 \\
\hline
\end{tabular}

Table XI: Comparing Voxel S values of Radionuclide: Sr89 with Voxel size $6 \mathrm{~mm}$ using Monte Carlo Volume Integration (my method) and Monte Carlo Radiation Transport Simulation (Bolch Method) 
Distance is in $\mathrm{mm}$.

Svalues are in $\mathrm{mGy} / \mathrm{Mbq}$-s.

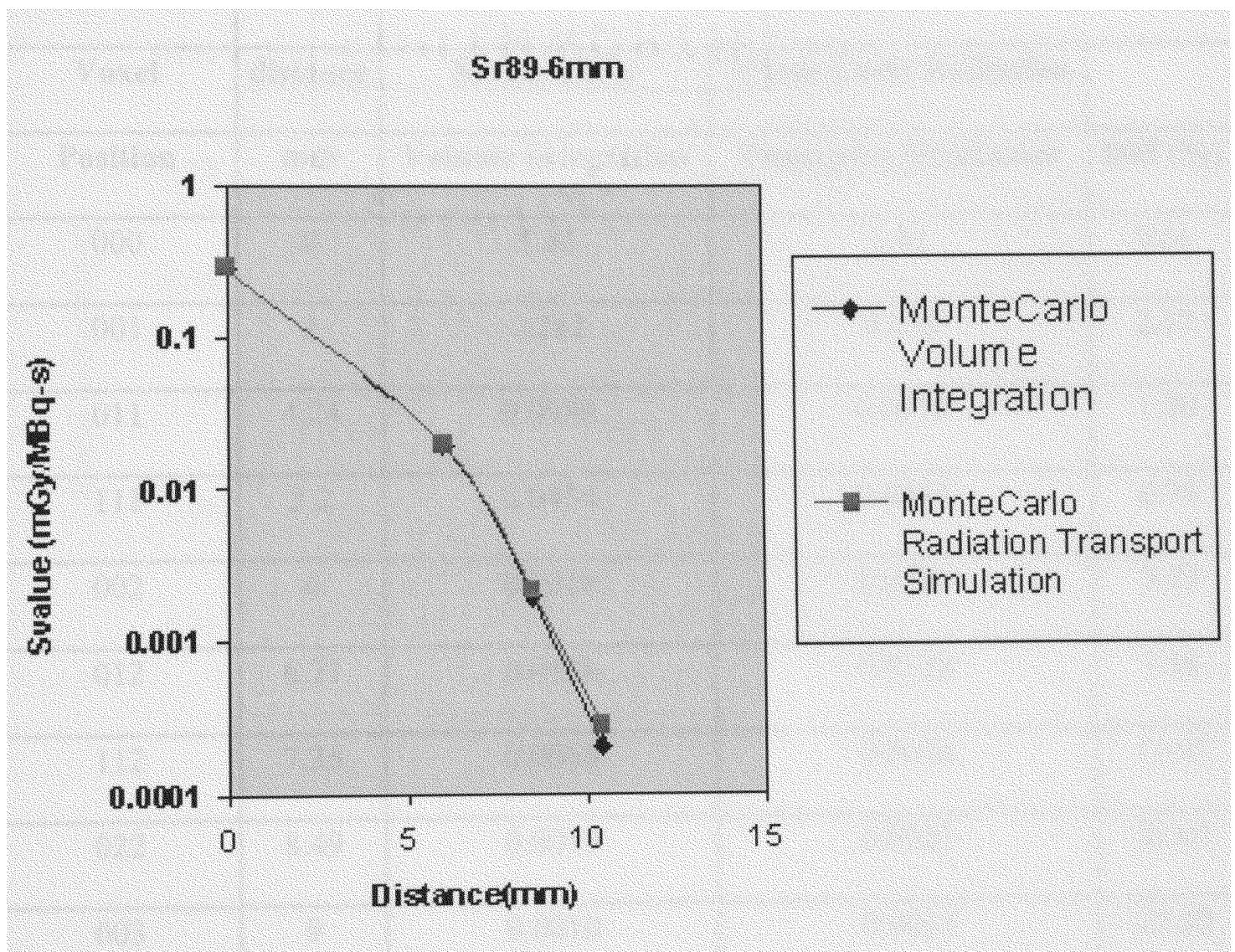

Figure 4: Comparing Voxel S values of Radionuclide: Sr89 with Voxel size 6mm using Monte Carlo Volume Integration (my method) and Monte Carlo Radiation Transport Simulation (Bolch Method) 
Distance is in $\mathrm{mm}$.

Svalues are in $\mathrm{mGy} / \mathrm{Mbq}-\mathrm{s}$.

\begin{tabular}{|c|c|c|c|c|}
\hline Radionuclide & $Y 90$ & & & \\
\hline Voxel Size & $3 \mathrm{~mm}$ & & & \\
\hline Voxel & distance & Monte Carlo & Monte Carlo Radiation & \\
\hline Position & $\mathbf{m m}$ & Volume Integration & Transport Simulation & Diff $(\%)$ \\
\hline 000 & 0 & 1.62 & 1.61 & 0.61 \\
\hline 001 & 3 & 0.282 & 0.276 & 2.17 \\
\hline 011 & 4.24 & 0.0988 & 0.0976 & 1.20 \\
\hline 111 & 5.2 & 0.0450 & 0.0453 & 0.74 \\
\hline 002 & 6 & 0.0218 & 0.0226 & 3.72 \\
\hline 012 & 6.71 & 0.0118 & 0.0128 & 7.48 \\
\hline 112 & 7.35 & 0.0065 & 0.0074 & 11.92 \\
\hline 022 & 8.49 & 0.0019 & 0.0025 & 22.63 \\
\hline 003 & 9 & 0.0010 & 0.0013 & 27.02 \\
\hline 013 & 9.49 & 0.0005 & 0.0008 & 34.51 \\
\hline 113 & 12.8 & 0.0003 & 0.0004 & 39.53 \\
\hline
\end{tabular}

Table XII: Comparing Voxel S values of Radionuclide: Y90 with Voxel size $3 \mathrm{~mm}$ using Monte Carlo Volume Integration (my method) and Monte Carlo Radiation Transport Simulation (Bolch Method) 
Distance is in $\mathrm{mm}$.

Svalues are in $\mathrm{mGy} / \mathrm{Mbq}-\mathrm{s}$.

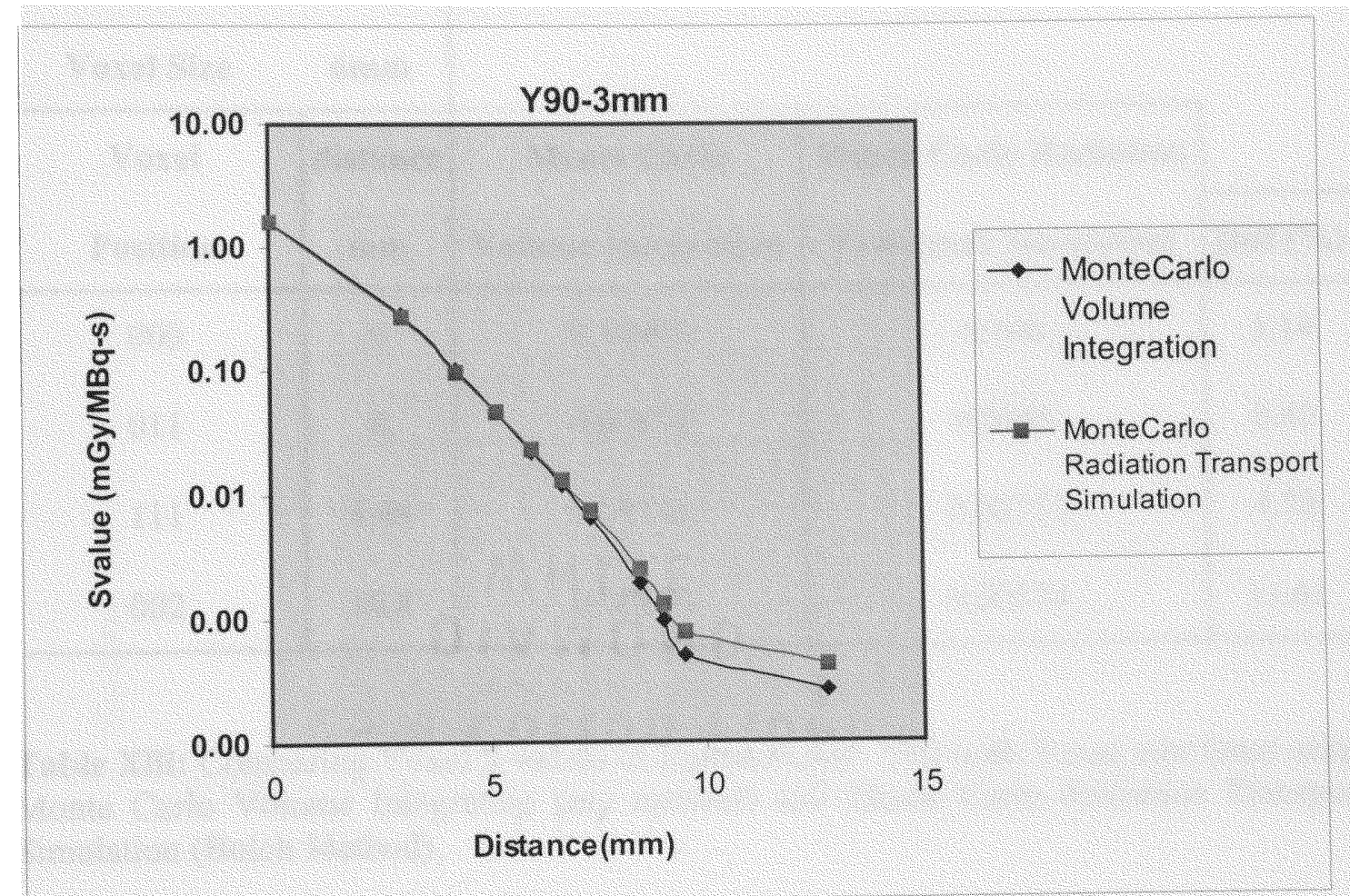

Figure 5: Comparing Voxel S values of Radionuclide: Y90 with Voxel size $3 \mathrm{~mm}$ using Monte Carlo Volume Integration (my method) and Monte Carlo Radiation Transport Simulation (Bolch Method) 
Distance is in $\mathrm{mm}$.

Svalues are in $\mathrm{mGy} / \mathrm{Mbq}-\mathrm{s}$.

\begin{tabular}{|c|c|c|c|c|}
\hline $\begin{array}{c}\text { Radionuclide } \\
\text { Voxel Size }\end{array}$ & \multicolumn{5}{|c|}{ Y90 } \\
6oxm & distance & Monte Carlo & Monte Carlo Radiation & \\
\cline { 4 - 5 } Position & $\mathbf{m m}$ & Volume Integration & Transport Simulation & Diff (\%) \\
\hline 000 & 0 & 0.34995 & 0.346 & 1.14 \\
011 & 6 & 0.03934 & 0.0395 & 0.42 \\
111 & 8.48 & 0.00721 & 0.00757 & 4.78 \\
002 & 10.4 & 0.00154 & 0.00174 & 11.61 \\
\hline
\end{tabular}

Table XIIl: Comparing Voxel S values of Radionuclide: Y90 with Voxel size $6 \mathrm{~mm}$ using Monte Carlo Volume Integration (my method) and Monte Carlo Radiation Transport Simulation (Bolch Method) 
Distance is in $\mathrm{mm}$.

Svalues are in $\mathrm{mGy} / \mathrm{Mbq}-\mathrm{s}$.

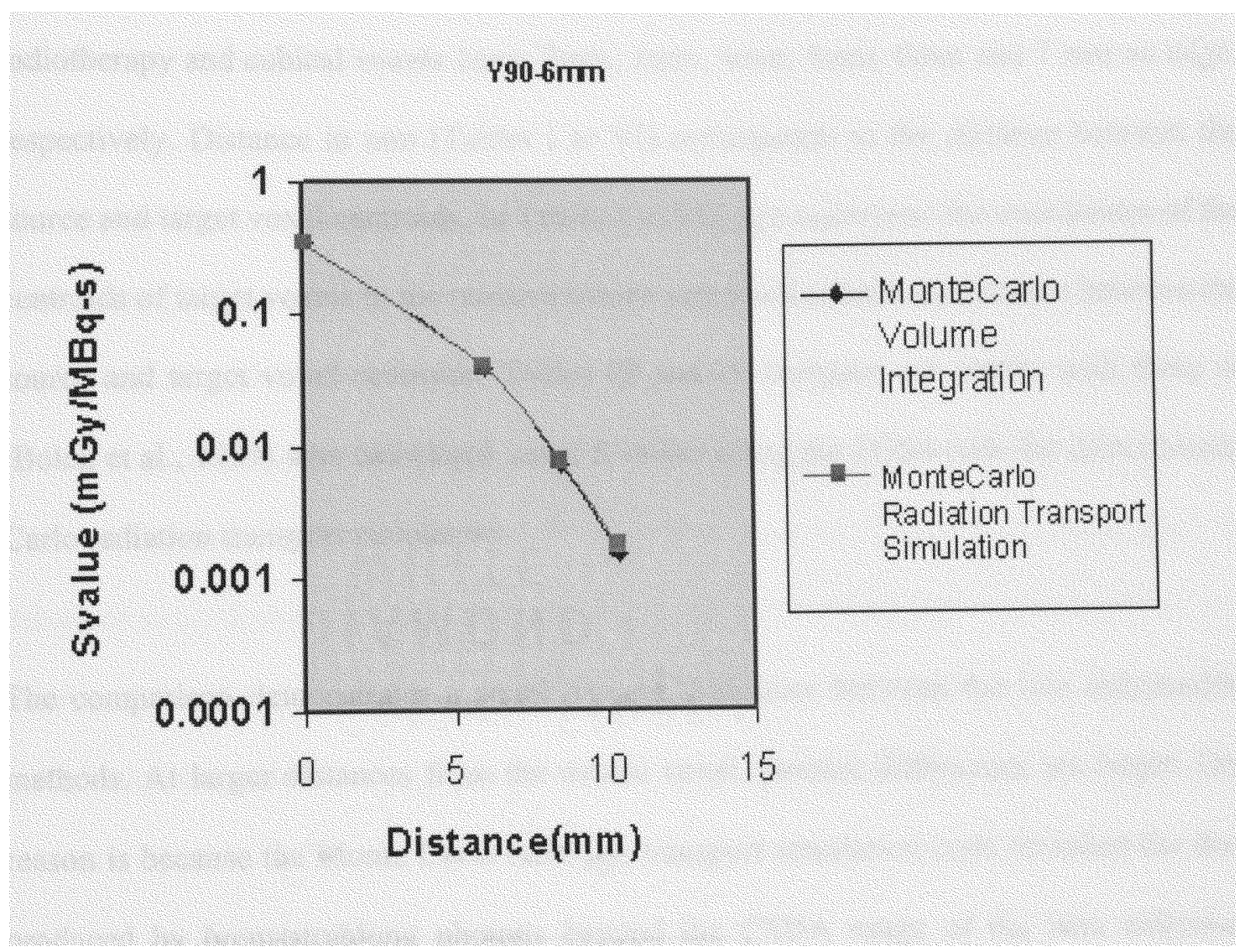

Figure 6: Comparing Voxel S values of Radionuclide: Y90 with Voxel size 6mm using Monte Carlo Volume Integration (my method) and Monte Carlo Radiation Transport Simulation (Bolch Method) 


\section{DISCUSSION AND CONCLUSIONS}

\subsection{Discussion}

Tables I to VI show the software output for 10 radionuclides of interest in internal radiotherapy and cubical voxels $1 \mathrm{~mm}, 2 \mathrm{~mm}, 3 \mathrm{~mm}, 4 \mathrm{~mm}, 5 \mathrm{~mm}, 6 \mathrm{~mm}$ and $7 \mathrm{~mm}$ on edge, respectively. Distance in $\mathrm{mm}$ (Tables I to VI) corresponds to the distance between the source and target voxel centroids. In Tables I to VII, xyz represents the coordinates of the centroids of target voxels in the positive octant expressed as units of distance between the source and target voxel centroids. Tables III and IV compare our results with those of (Bolch et al., 1999) who calculated voxel S values using the EGS4 code for direct Monte Carlo radiation transport simulation.

The comparison demonstrates a small percent difference between the two independent methods. At larger distances from the source voxel, percent differences are larger. One reason is because the Monte Carlo radiation transport simulation code included the dose produced by bremsstrahlung photons beyond the CSDA range of the beta emissions (Bolch. Et.al., 1999). Other additional reasons are the increased statistical uncertainty of dose calculations by both methods at large distances from the source (lower number of simulated rays) and different algorithms for simulation of radiation transport and energy deposition (Bolch et.al., 1999; Cross et.al., 1992). While Bolch et al (Bolch. Et.al., 1999) used the PRESTA algorithms for direct Monte Carlo simulations in tissue and calculate the electron dose to distances approximating the CSDA range of the electron energy, the dose-point kernels of Cross (Cross et.al., 1992) were calculated in water using the 
ACCEPT code with a cut-off energy of $2 \mathrm{keV}$ or somewhat more for high initial energies. However, at those larger distances from the source voxel the contribution to the absorbed dose can be considered negligible. Since direct Monte Carlo radiation transport simulation is considered the theoretical gold standard for radiation dosimetry, Tables III and IV show the validity of our algorithm.

The Monte Carlo volume integration of dose point-kernels presented in this report has been already suggested by Bolch (Bolch et.al., 1999). Our contribution has been to implement a valid algorithm in computationally efficient software and verify the accuracy of calculations by comparing our results with those determined by direct Monte Carlo radiation transport simulation. The software can be used by any laboratory to calculate voxel $\mathrm{S}$ values of beta emissions from tabulated dose point-kernels and for any combination of pixel edges and thickness of SPECT and PET images without the complexity and expertise needed for direct Monte Carlo radiation transport simulations. 


\section{Conclusions:}

- The comparison demonstrates a small percentage difference between the two independent methods.

- When distance is less, the error in calculating dose is less than $10 \%$.

- At larger distances from the source voxel, percentage differences are larger. But at larger distances the dose rates are very less and those values are negligible.

- Though this method has error at large distances, it is useful to calculate voxelSvalues for all cubical and non-cubical voxels and thickness and for all coordinates of SPECT and PET images with less time and without the complexity of direct Monte Carlo radiation transport simulations.

- This method is very fast and it takes minutes to run this program. 


\section{REFERENCES}

1. Darrell R.Fisher, Internal Dosimetry for systemic Radiation Therapy, Vol 10, No 2 (April)2000:pp 123-132, Seminars in Radiation Oncology.

2. Pat.B. Zanzonico, Internal Radionuclide Radiation Dosimetry : A Review of Basic Concepts and Recent Developments, J.Nucl.Med 2000; 41:297-308.

3. Timothy k.Johnson, David Mc Clure, and Steven Mc Court, MABDOSE1. Charecterisation of a general purpose dose estimation code : J.Med.Phys.Vol.26 No.7,July 1999.

4. Johnson, T.K., Mc clure,D.,Mc Court, S.(1999b),MABDOSE II: validation of a general purpose dose estimation code. Med. Phys.26: 1396-1403.

5. Evelyn E.Watson and Michael G.Stabin, Jeffry A. Siegel, "MIRD formulation", J. Med.Phys., Vol.20, No.2, Pt.2, Mar/Apr 1993

6. R. Loevinger, T. F. Budinger, and E. E. Watson, "MIRD Primer for Absorbed Dose Calculations (Society of Nuclear Medicine, New York, NY, 1988).

7. Loevinger, R., Budinger, T., Watson, E., 1988. MIRD Primer for Absorbed Dose Calculations. Society of Nuclear Medicine.

8. Johnson, T. K., 1988. MABDOSE: a generalized program for internal radionuclide dosimetry. Comput. Methods Programs Biomed. 27, 159-167.

9. Kolbert, K. S., Sgouros, G., Scott, A. M., Bronstein, J. E., Malane, R. A., Zhang, J. J., Kalaigian, H., Mcnamara, S., Schwartz, L. and Larson, S. M., 1997. Implementation and evaluation of patient-specific three-dimensional internal dosimetry. J. Nucl. Med. 38, pp. 301-308.

10. Erdi, A. K., Erdi, Y. E., Yorke, E. D. and Wessels, B. W., 1996. Treatment planning for radio-immunotherapy. Phys. Med. Biol. 41, pp. 2009-2026.

11. Berger, M., 1973. Improved point kernels for electron and beta ray dosimetry. Center for Radiation Research, Department of Commerce, Washington D.C., U.S.A

12. Prentice, W. V., Nunes, J. and Kwok, C. S., 1989. Beta dose point kernels for radionuclides of potential use in radioimmunotherapy. J. Nucl. Med. 30, pp. 10361046.

13. Simpkin, D. J. and Mackie, T. R., 1990. EGS4 Monte Carlo determination of the beta dose kernel in water. Med. Phys. 17, pp. 179-186. 
14. Leichner, P. K., 1994. A unified approach to photon and beta particle dosimetry. $J$. Nucl. Med. 35, pp. 1721-1729.

15. Sgouros, G., 1993. Bone marrow dosimetry in radioimmunotherapy: theoretical considerations. J. Nucl. Med. 34, p. 689.

16. Giap, H. B., Macey, D. J., Poduloff, D. A. and Boyer, A. L., 1994. Development of a SPECT-based 3D methodology for internal dosimetry of radioimmunotherapy. $J$. Nucl. Med. 35, p. 123P.

17. Akabani, G., Hawkins, W. G., Eckblade, M. B. and Leichner, P. K., 1997. Patientspecific dosimetry using quantitative SPECT imaging and three-dimensional discrete fourier transform convolution. J. Nucl. Med. 38, pp. 308-314

18. Raeside, D. E., 1976. Monte Carlo principles and applications. Phys. Med. Biol. 21, pp. 181-197

19. Simpkin, D. J. and Mackie, T, R., 1990. EGS4 Monte Carlo determination of the beta dose kernel in water. Med. Phys. 17, pp. 179-186

20. Briesmeister, J., 1993. MCNP: A general Monte Carlo n-particle transport code. MCNP User's Manual. Los Alamos National Laboratory

21. Furhang, E. E., Chui, C. S., Kolbert, K. S., Larson, S. M. and Sgouros, G., 1997. Implementation of a Monte Carlo dosimetry method for patient-specific internal emitter therapy. Med. Phys. 24, pp. 1163-1172

22. W.G.Cross, N.O.Freedman, and P.Y.Wong, Tables of Beta-Ray Dose Distributions in Water (Atomic Energy of Canada, Ltd., Report No. AECL-10521, Ontario, Canada, 1992).

23. W.E.Bolch,L.G.Bouchet, J.S. Robertson, B.W. Wessels, J.A.Siegel,R.W.Howell, A.D.Erdi,B.Aydogan,S.Costes, and E.E.Watson,"MIRD Pamphlet No.17: the dosimetry of nonuniform activity distributions-radionuclide $S$ values at the voxel level", J.Nucl.Med.40:11S-36S(1999).

24. B.W. Wessels, and J.A. Siegel, "Dosimetry overview: keeping score on the scorekeepers", Cancer 80 (Suppl): 2501 - 2504 (1997).

25. R.W. Howell, B.W. Wessels, and R. Loevinger, "The MIRD perspective 1999", J. Nucl. Med., 40: 3S - 10S (1999).

26. W.E. Bolch, L.G. Bouchet, J.S. Robertson, B.W. Wessels, J.A. Siegel, R.W. Howell, A.K. Erdi, B. Aydogan, S. Costes, and E.E. Watson, "MIRD Pamphlet No. 17: The 
dosimetry of nonuniform activity distributions-radionuclide $\mathrm{S}$ values at the voxel level", J. Nucl. Med. 40: 11S - 36S (1999).

27. Liu, L.E. Williams, J.Y. Wong, and A.A. Raubitschek, "Monte Carlo assisted voxel source kernel method (MAVSK) for internal beta dosimetry", Nucl. Med. Biol., 25: 423 - 433 (1998).

28. W.G. Cross, N.O. Freedman, and P.Y. Wong, Tables of Beta-Ray Dose Distributions in Water (Atomic Energy of Canada, Ltd., Report No. AECL-10521, Ontario, Canada, 1992).

29. Clairand, I., Bouchet, L.G., Richard, M., Durigon, M., Di Paola, M., Aubert, B. (2000), Improvement of internal dose calculations using mathematical models of different adult heights. Physics in Medicine and Biology, 45: 2771-2785.

30. Furhang, E.E., Sgouros G., Chui, C.S. (1996), Radionuclide photon dose kernels for internal emitter dosimetry. Med. Phys. 23: 759-764.

31. Furhang, E.E., Chui, C.S., Kolbert, K.S.et al.(1997), Implementation of a Monte Carlo dosimetry method for patient-specific internal emitter therapy. Med. Phys.24: 1163-1172. 


\section{APPENDICES}

\section{APPENDIX-I}

\section{Creation of Random Numbers: (Program)}

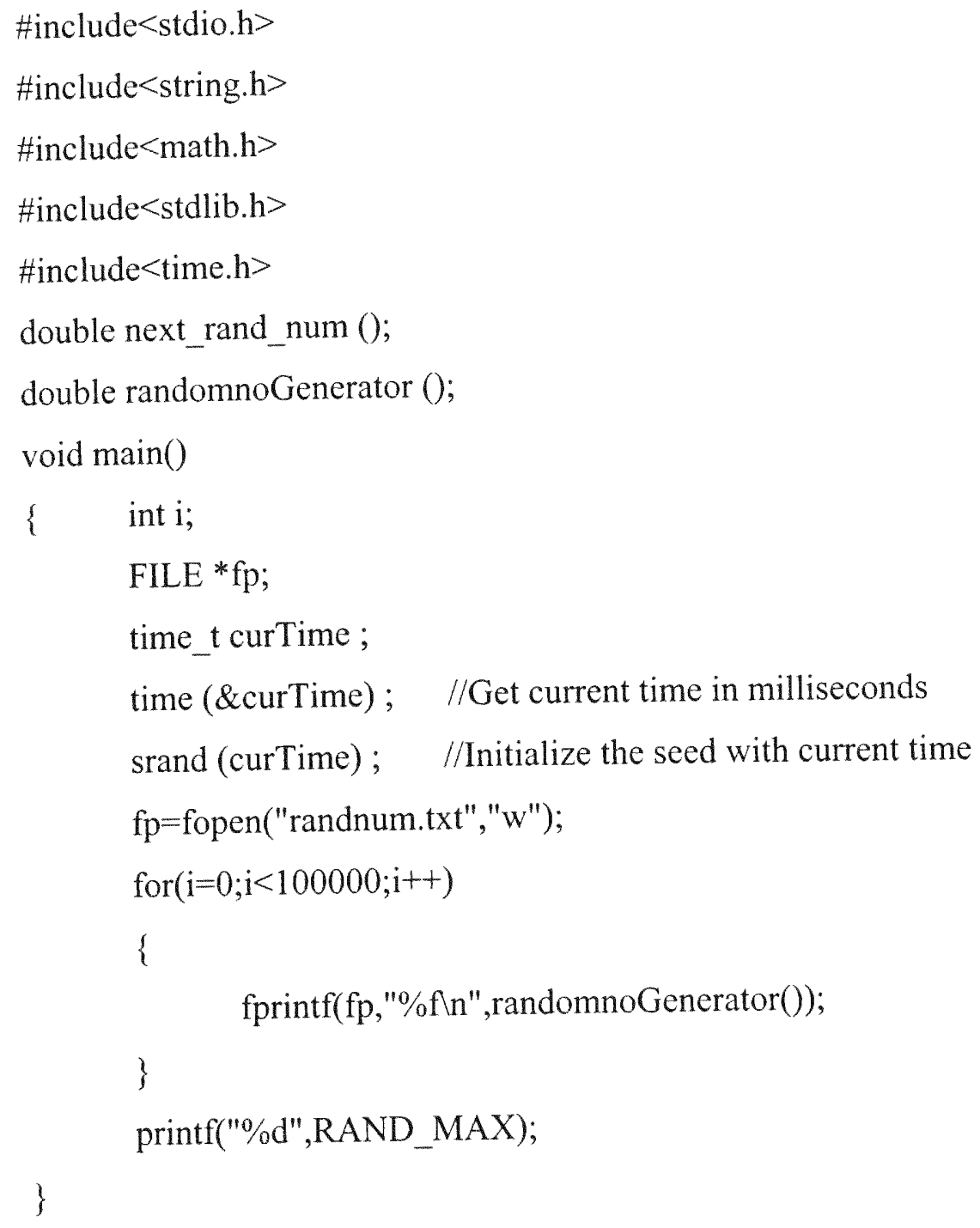




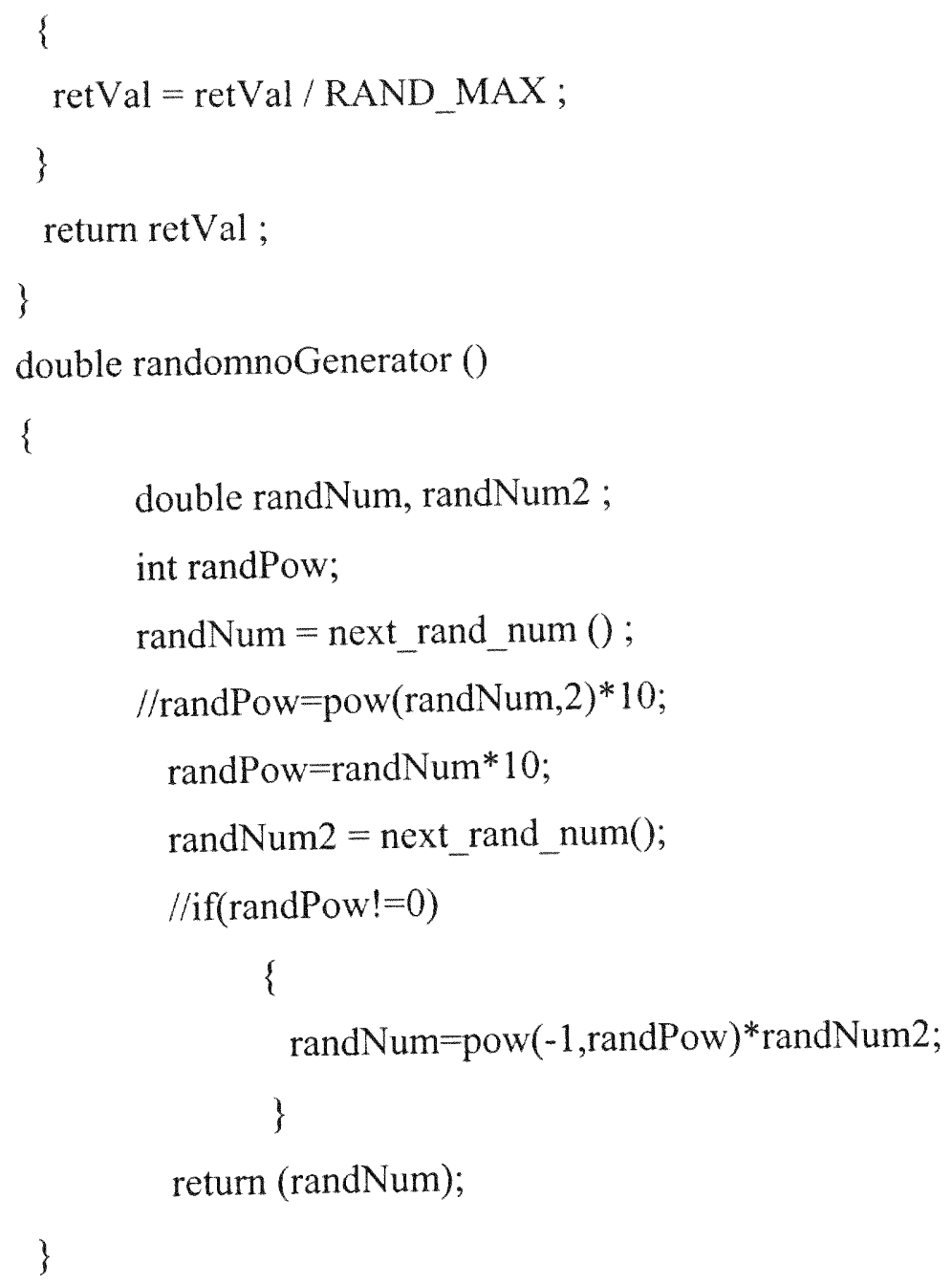

\} 
Calculation of Voxel Svalues (Program code to Calculate Svoxel Values)

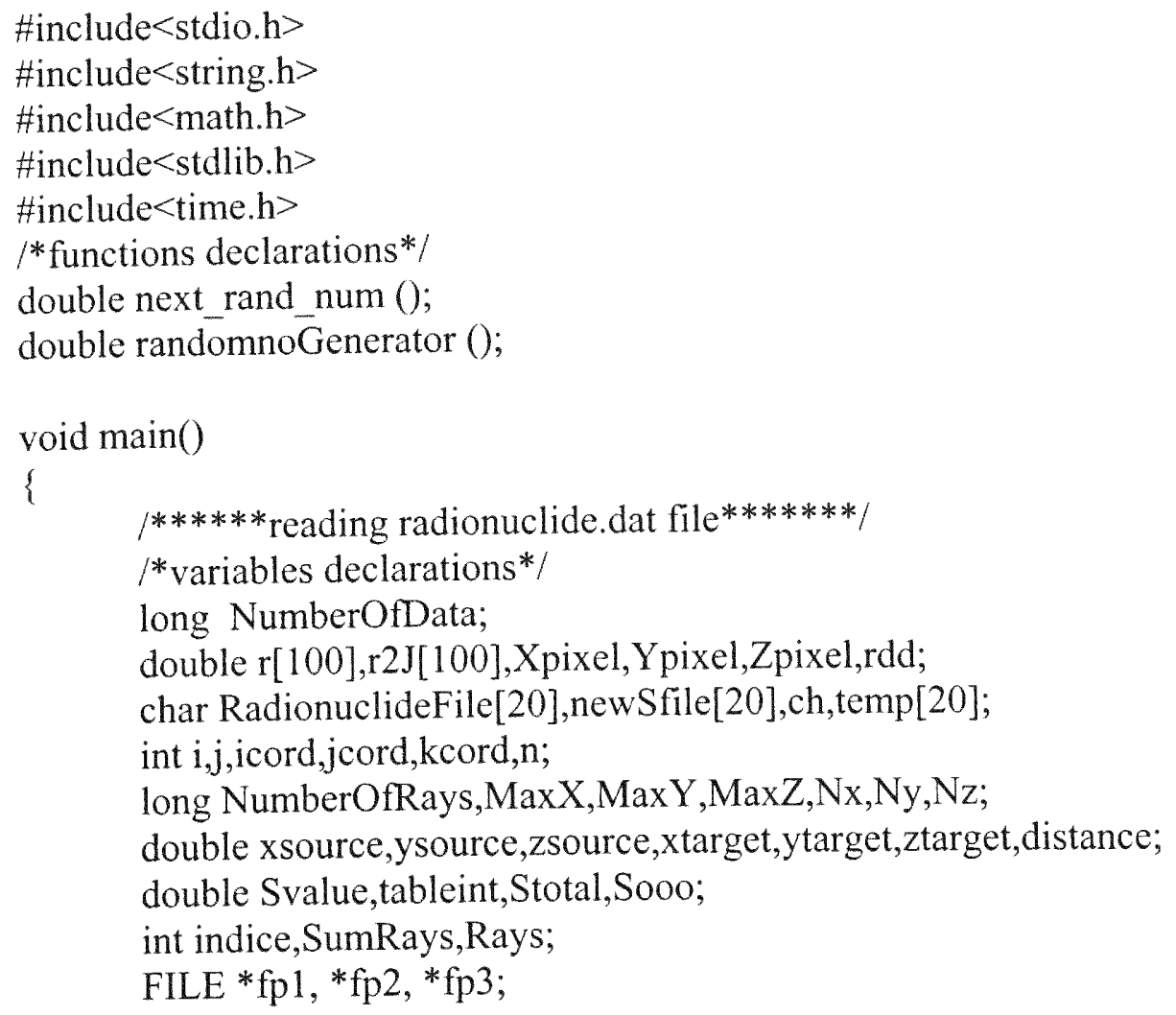

$/ /$ Get current time in milliseconds

time_t curTime;

//Initialize the seed with current time

time (\&curTime) ;

srand (curTime);

printf("enter the radionuclide.dat file eg:Cu64.dat $\backslash n ")$; scanf("\%s",RadionuclideFile);

printf("enter the new S-file $\backslash n "$ );

scanf("\%s",newSfile);

$\mathrm{fpl}$ =fopen(RadionuclideFile, $" \mathrm{r} "$ );

if $(\mathrm{fp} 1==$ NULL $)$

\{

$$
\begin{aligned}
& \text { printf("\ncannot open file } \backslash n ") \text {; } \\
& \text { exit(1); }
\end{aligned}
$$

)

fseek(fp1,5,0);

$/ /$ the number of data points are read into a variable NumberOfData. 
fscanf(fp1,"\%ld",\&NumberOfData);

$\mathrm{r} 2 \mathrm{~J}[]$

$/ /$ the values from the first and second column are read and stored in arrays $r[]$ and

//respectively.

for $(j=0 ; j<$ NumberOfData $;++)$

\{

$$
\begin{aligned}
& \mathrm{i}=0 ; \\
& \text { while }\left((\operatorname{ch}=\operatorname{fgetc}(\operatorname{fp} 1)) !=^{\prime},{ }^{\prime}\right)
\end{aligned}
$$

\{

temp[i] $=\mathrm{ch}$;

$$
\text { i++; }
$$

temp[i] ${ }^{3} \backslash 0^{\prime}$;

$\mathrm{r}[\mathrm{i}]=\operatorname{atof}(\mathrm{temp})$

fscanf(fpl,"\%lf",\&r2J[j]);

$\mathrm{r} 2 \mathrm{~J}[\mathrm{j}]=\mathrm{r} 2 \mathrm{~J}[\mathrm{j}] * 0.0002778$;

fclose(fp1);

$/ / * * * * * * * * * * *$ reading data from voxel.data

fp2=fopen("voxel.dat","r");

if $(\mathrm{fp} 2==\mathrm{NULL})$

\{

printf("\ncannot open file $\backslash n ")$;

exit(1);

\}

//the data read from the file are stored in the corresponding variables.

while $\left((\mathrm{ch}=\text { fgetc }(\mathrm{fp} 2)) !^{\prime}=^{\prime \prime}:\right)$;

fscanf(fp2,"\%lf",\&Xpixel);

while $\left((\mathrm{ch}=\right.$ fgetc $($ fp2 $\left.)) !=^{\prime \prime}: '\right)$;

fscanf(fp2,"\%lf",\&Ypixel);

while $\left((\mathrm{ch}=\right.$ fgetc $\left.(\mathrm{fp} 2)) !=^{\prime}:{ }^{\prime}\right)$;

fscanf(fp2,"\%lf",\&Zpixel);

while((ch=fgetc(fp2))! =':');

fscanf(fp2,"\%ld",\&NumberOfRays);

while $((\mathrm{ch}=$ fgetc(fp2))! =':');

fscanf(fp2,"\%ld",\&MaxX); 


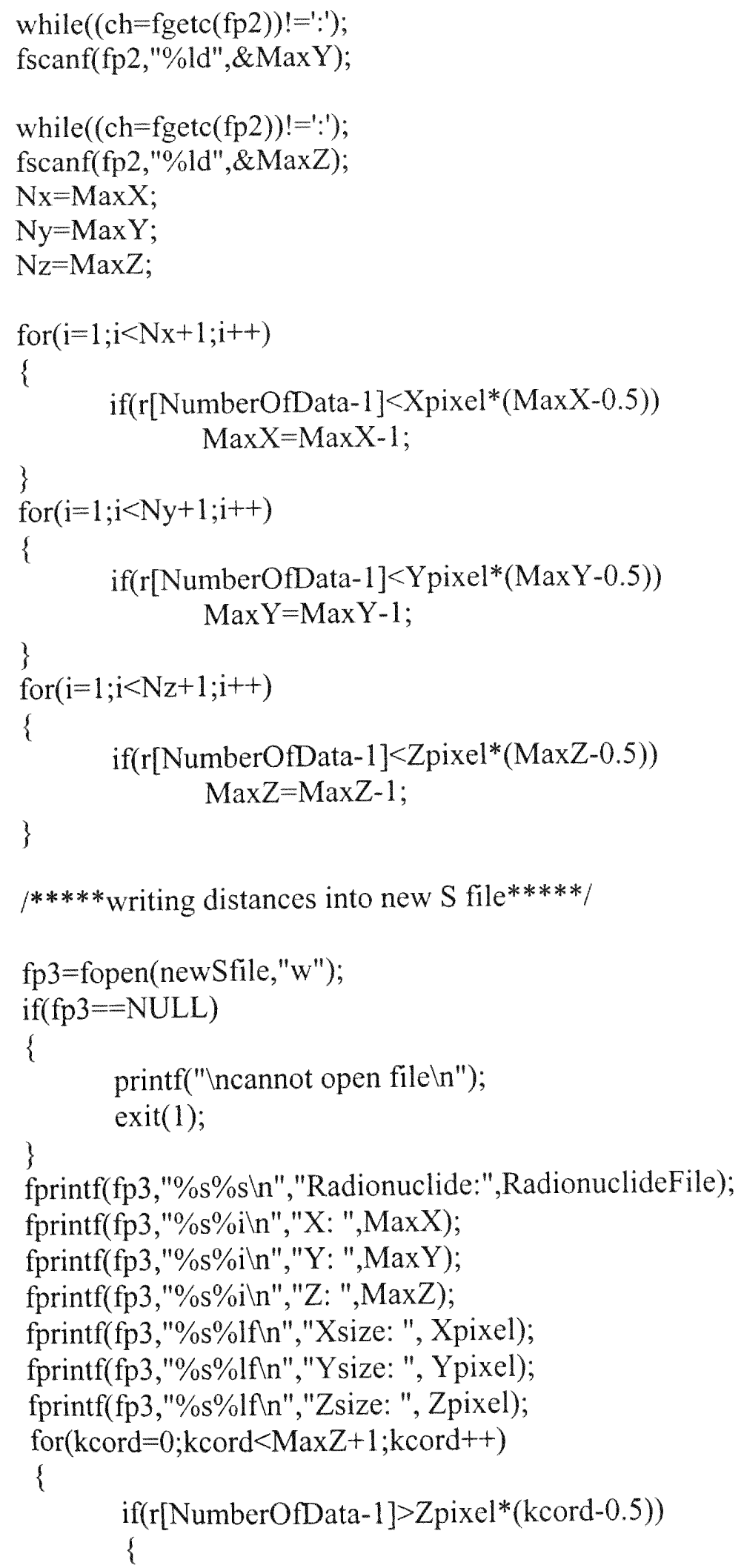


for $($ jcord $=\mathrm{kcord}$; $\operatorname{jcord}<\mathrm{MaxY}+1$; jcord ++ )

\{

if $\left(r[\right.$ NumberOfData-1 $]>$ Ypixel $^{*}($ jcord-0.5))

for $($ icord $=$ jcord; icord $<$ Max $\mathrm{X}+1$; icord ++ )

\{

1] $>$ Xpixel*(icord-0.5))

if(r[NumberOfData-

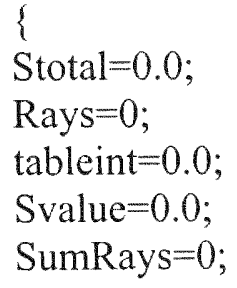

for $(\mathrm{n}=0 ; \mathrm{n}<$ NumberOfRays; $\mathrm{n}++)$

rdd=randomnoGenerator();

xsource=randomnoGenerator ()$^{*}(\mathrm{Xpixel} / 2.0)$;

ysource $=$ randomnoGenerator ()$^{*}($ Ypixel/2.0);

zsource $=$ randomnoGenerator ()$^{*}($ Zpixel/2.0);

xtarget=randomnoGenerator ()$^{*}(\mathrm{Xpixel} / 2.0)$;

ytarget=randomnoGenerator()*(Ypixel/2.0);

ztarget=randomnoGenerator( $)^{*}($ Zpixel/2.0);

xtarget=icord*(Xpixel) + xtarget;

ytarget $=$ jcord*(Ypixel $)+y \operatorname{target}$;

ztarget $=$ kcord*(Zpixel) + ztarget;

distance= pow $($ xsource-xtarget, 2.0$)+$ pow $($ ysource-ytarget, 2.0$)+$ pow $(z$ sourceztarget,2.0);

distance $=$ pow $($ distance, 0.5$)$; 
if $($ distance $<\mathrm{r}[1] / 100.0)$

distance $=r[1] / 100.0$

for $(\mathrm{i}=0 ; \mathrm{i}<$ NumberOfData $-1 ; \mathrm{i}++)$

if $($ distance $>r[i] \& \&$ distance $<r[i+1])$

indice $=\mathrm{i}$;

if(distance $>$ r[NumberOfData-1])

indice $=$ NumberOfData- 1 ;

if(indice $<$ NumberOfData-1)

tableint $=((\log (\mathrm{r} 2 \mathrm{~J}[$ indice $])-\log (\mathrm{r} 2 \mathrm{~J}[$ indice +1$])) *(\mathrm{r}[$ indice $]$-distance $)) /(\mathrm{r}[$ indice $]-$ $\mathrm{r}$ [indice +1$]$ );

Svalue $=\exp (\log (\mathrm{r} 2 \mathrm{~J}[$ indice $])$-tableint $)$

Svalue $=$ Svalue $/\left(\right.$ distance ${ }^{*}$ distance $)$

Stotal $=$ Stotal + Svalue;

Rays $=$ Rays +1 ;

SumRays $=$ SumRays +1 ;

simulation 
if $($ SumRays $>0)$ Svalue $=$ Stotal $/$ SumRays;

$\& \&$ jcord $==0 \& \&$ kcord $==0)$ Sooo $=$ Svalue;

if $($ icord $==0$

if

(Svalue $>0.0001 *$ Sooo)

printf("\%d\%d\%d\%s \%fln",icord,jcord,kcord,":",Svalue);

fprintf(fp3,"\%d\%d\%d\%s \%fn",icord,jcord,kcord,":",Svalue);

simulation

\}//end of $\mathrm{n}$-th

\}//end od if $\mathrm{r}<\mathrm{icord}$

\}//end of icord

$3 / /$ end of if $r<j$ cord

\}//end of jcord

\}//end of if $\mathrm{r}<\mathrm{kcord}$

\}//end of kcord

fclose(fp3);

\}//end of main

$/ *$ functions' definitions*/

double next_rand_num ()

\{

double retVal ;

$\mathrm{ret} \mathrm{Val}=1.0 *$ rand () ;

$1 *$ adjusting the random numbers to less than $1 * /$

while $($ retVal $>1)$

\{

retVal = retVal $/$ RAND_MAX;

\}

return retVal ;

\}

double randomnoGenerator ()

\{

double randNum, randNum2 ;

int randPow; 
$1 *$ generating randomnumbers between -1 and $1 * /$

randNum = next rand_num ();

randPow $=$ randNum* 10 ;

randNum2 $=$ next_rand_num();

randNum=pow $(-1 \text {,randPow })^{*}$ randNum 2 ;

return (randNum);

) 